\title{
Orientifolds, RR torsion, and K-theory
}

\section{Oren Bergman}

California Institute of Technology, Pasadena CA 91125, USA and

CIT/USC Center for Theoretical Physics

Univ. of Southern California, Los Angeles CA, USA

E-mail: 'bergman@theory.caltech.edü'

\section{Eric Gimon}

School of Natural Sciences, Institute for Advanced Study

Einstein Drive, Princeton NJ 08540, USA

E-mail: 'gimon@isns.ias.edu'

\section{Shigeki Sugimoto}

CIT/USC Center for Theoretical Physics, Univ. of Southern California

Los Angeles CA, USA

E-mail: 'sugimoto@citusc.usc.edù'

Abstract: We analyze the role of $R R$ fluxes in orientifold backgrounds from the point of view of K-theory, and demonstrate some physical implications of describing these fluxes in K-theory rather than cohomology. In particular, we show that certain fractional shifts in RR charge quantization due to discrete $R R$ fluxes are naturally explained in K-theory. We also show that some orientifold backgrounds, which are considered distinct in the cohomology classification, become equivalent in the K-theory description, while others become unphysical.

Keywords: 'D-branes, Differential and Âlgebraic Geometry'. 


\section{Contents}

ii. Introduction

2. Review of orientifolds

'2.1' Basics

12.21 Cohomology torsion variants

2.3. Brane realization of torsion

3. Orientifolds in K-theory

'3.1' Real K-theory

RR fields in orientifold backgrounds

13.3. Comparing with cohomology

每. Applications

'A. $\overline{1}$ ' Charge quantization

Equivalent orientifolds

Unphysical orientifolds

75. The orientifold 6-plane

15.1' K-theory

Anomalies

15.3. Components of type-I vacuua

6. Conclusions and open questions

Aي.'. AHSS in complex and real K-theory

:Ä. I' Defining the associated graded complex

A $\overline{2}$ Computing GrK using a spectral sequence

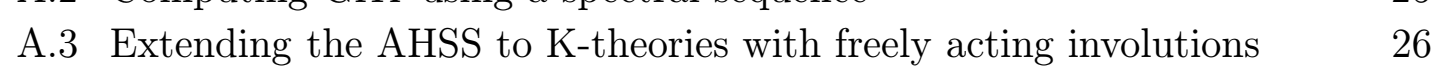

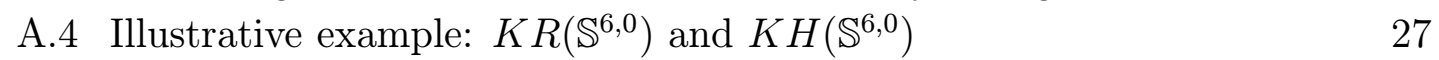

\section{Introduction}

Orientifold planes are intriguing objects in many ways. They appear, at least perturbatively, to be void of dynamics, and in this respect they are similar to orbifolds. On the other hand orientifold planes are also similar to D-branes, in that they appear with the same dimensionalities, carry RR charge, and have a non-vanishing tension. 


\begin{tabular}{|l|l|l|l|}
\hline$\left(H, G_{6-p}\right)$ & $\mathrm{O} p$ variant & $\mathrm{D} p$ gauge group & charge \\
\hline$(0,0)$ & $\mathrm{O} p^{-}$ & $\mathrm{SO}(2 n)$ & $-2^{p-5}$ \\
$(1 / 2,0)$ & $\mathrm{O} p^{+}$ & $\mathrm{U} \mathrm{Sp}(2 n)$ & $+2^{p-5}$ \\
$(0,1 / 2)$ & $\widetilde{\mathrm{O} p}^{-}$ & $\mathrm{SO}(2 n+1)$ & $-2^{p-5}+1 / 2$ \\
$(1 / 2,1 / 2)$ & $\widetilde{\mathrm{O} p}^{+}$ & $\mathrm{U} \mathrm{Sp}(2 n)$ & $+2^{p-5}$ \\
\hline
\end{tabular}

Table 1: Orientifold plane variants and their charges.

However the latter two differ from their D-brane values in two important ways. In some cases the tension of an orientifold plane is negative, and in some cases its charge (and tension) is fractional.

There are actually a variety of orientifold planes for each dimensionality $p$, associated with the possibility of turning on discrete fluxes of certain anti-symmetric tensor fields [i, in three-form $H$, and the $\mathrm{RR}(6-p)$-form $G_{6-p}$, and have traditionally been classified by the (integral) cohomologies $H^{3}\left(\mathbb{R P}^{8-p}\right)$ and $H^{6-p}\left(\mathbb{R P}^{8-p}\right)$, respectively. The space $\mathbb{R P}^{8-p}$ is the "sphere" that surrounds the $\mathrm{O} p$-plane in the reduced space of the orientifold projection. Except for the case $p=6$, both of these cohomologies are $\mathbb{Z}_{2}$, so the fluxes are torsion classes. This means that there are four variants of the orientifold plane for each $p$ (for $p \leq 5$ ), depending on the values of the discrete fluxes (table 1 ). For $p<2$ there are actually additional variants, which are associated with a discrete $G_{2-p}$-flux [2i] .

One immediately observes that orientifold planes with $p \leq 4$ are fractionally charged (relative to D $p$-branes). This appears to be at odds with Dirac's quantization condition, suitably generalized to higher rank anti-symmetric tensor fields, or equivalently with the idea that $\mathrm{RR}$ charges and fields take values in integral cohomology. In particular, since the $\mathrm{O} p$-plane is charged under $C_{p+1}$, the relevant cohomology group is given by $H^{8-p}\left(\mathbb{R P}^{8-p}\right)=\mathbb{Z}$. The generator of this group has a unit $\mathrm{D} p$-brane charge, so the $\mathrm{O} p$-plane charge $\pm 2^{p-5}$ is not an allowed value for $p \leq 4$. In addition, the presence of $\mathrm{RR}$ torsion in $H^{6-p}\left(\mathbb{R P}^{8-p}\right)$ shifts the charge by $1 / 2$ for the $\mathrm{O}^{-}$plane, but not for the $\mathrm{O} p^{+}$plane. So in the first case it seems to lead to a further violation of Dirac's condition.

In some cases the violation can be attributed to an anomaly in the path integral for the dual magnetic object, i.e. a $\mathrm{D}(6-p)$-brane, and can be expressed in terms of spacetime fields [3제] of the $\mathrm{O} 4^{ \pm}$plane is consistent with an anomalous phase in the D2-brane path integral coming from the fermion determinant [i] to the phase in order to explain the 0 charge of the $\widetilde{\mathrm{O}}^{-}$variant, and yet another to explain the $+1 / 2$ charge of $\widetilde{\mathrm{O}}^{+}$. One could imagine that the latter is due to the term $\int B \wedge C_{1}$ in the D2-brane action, but there is no obvious candidate for the former contribution. For other orientifold planes one does not even have an analog of the anomaly contribution of [3]. 
All this indicates that integral cohomology is not the appropriate mathematical framework for RR fields. We are familiar with the idea that D-branes, or more properly $\mathrm{RR}$ charges, take values in K-theory rather than in integral cohomology

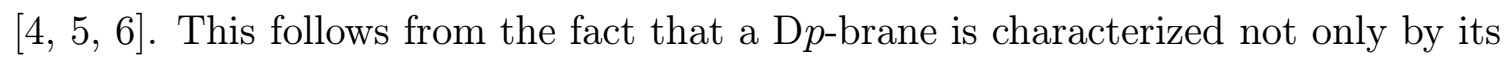
charge under the RR field $C_{p+1}$, but also by a gauge bundle, which in turn determines its charges under lower rank RR fields. It has recently been suggested that the RR fields take values in K-theory as well [0] created by D-brane sources, since the field at infinity should belong to the same class as the source which creates it. But the idea is that all RR fields, including source-free RR fluxes, are classified in K-theory.

In this paper we investigate some of the consequences of describing RR fluxes in K-theory rather than integral cohomology. In particular, this provides a partial explanation of the observed fractional charges; K-theory correlates different RR fields in such a way, that the discrete flux of $G_{6-p}$ alters the quantization of $G_{8-p}$-flux. For $p=6$ the former $\left(G_{0}\right)$ is integral rather than discrete, but there is an analogous correlation between the parity of $G_{0}$ and the half-integral part of $G_{2}$. Thus we are able to compute the shift in the quantization condition of the RR charge due to the discrete RR flux. We are not able to establish the quantization condition for the absolute charges however. In comparing the K-theory classification with the cohomological one we also discover that some discrete fluxes are actually trivial, while others are obstructed. This has interesting physical implications concerning some of the lower orientifold planes.

The rest of the paper is organized as follows. In section $\underset{-1}{2}$ we review what is known about orientifold planes, and their classification in terms of discrete fluxes in cohomology. In section ${ }_{-1}^{n}$ wis we describe the necessary K-theory machinery required for describing RR fields in orientifold backgrounds, and compute the relevant groups. We also explain how to compare the K-theory results with cohomology using a real version of the Atiyah-Hirzebruch spectral sequence (AHSS), which is further elaborated in the appendix. In section 愿 we discuss three implications of the difference between K-theory and cohomology in orientifolds. The first concerns the question of proper RR charge quantization, the second is the identification in K-theory of certain orientifold variants which are distinct in cohomology, and the third is the dismissal of cer-

tain orientifold variants as unphysical. Section the orientifold 6-plane, which in many ways was the original motivation for this work.

\section{Review of orientifolds}

\subsection{Basics}

Orientifolds are defined by gauging a discrete symmetry in string theory which includes a reversal of the string world-sheet orientation (denoted $\Omega$ ). The loci of points 
which are fixed under this symmetry are called orientifold planes. In particular, an orientifold $p$-plane $(\mathrm{O} p)$ in type-II string theory is given by the $\mathbb{Z}_{2}$ projection

$$
\text { Op }: \quad \mathbb{R}^{1, p} \times \mathbb{R}^{9-p} / \mathcal{I}_{9-p} \Omega \cdot\left\{\begin{array}{lll}
1 & p=0,1 & \bmod 4 \\
(-1)^{F_{L}} & p=2,3 & \bmod 4,
\end{array}\right.
$$

where $p$ is even in IIA and odd in IIB. The presence of $(-1)^{F_{L}}$ for $p=2,3 \bmod 4$ can be understood from the requirement that the generator square to 1 on fermions. For example, $\left(\mathcal{I}_{2} \Omega\right)^{2}=-1$ on fermions, but $\left(\mathcal{I}_{2} \Omega(-1)^{F_{L}}\right)^{2}=\left(\mathcal{I}_{2} \Omega\right)^{2}(-1)^{F_{L}+F_{R}}=+1$. The action of the orientifold on the antisymmetric tensor fields is given by

$$
\begin{array}{cl}
B \longrightarrow-B & \\
C_{p^{\prime}} \longrightarrow+C_{p^{\prime}} & p^{\prime}=p+1 \bmod 4 \\
C_{p^{\prime}} \longrightarrow-C_{p^{\prime}} & p^{\prime}=p+3 \bmod 4 .
\end{array}
$$

In particular, the projection leaves the $\mathrm{D} p$-brane invariant, and in fact preserves the same 16 supersymmetries.

If we include $n \mathrm{D} p$-branes (and their images), there are two possible actions on the open string Chan-Paton factors [9.9]1,

$$
\lambda \rightarrow M^{-1} \lambda^{T} M, \quad M=\mathbb{I}_{2 n \times 2 n} \text { or }\left[\begin{array}{cc}
0 & i \mathbb{I}_{n \times n} \\
-i \mathbb{I}_{n \times n} & 0
\end{array}\right](\equiv \mathbb{J}) .
$$

In the first case the resulting gauge group is $\mathrm{SO}(2 n)$, and in the second case $\mathrm{U} \mathrm{Sp}(2 n)$. The corresponding Möbius strip amplitudes, and therefore also the vacuum $\mathbb{R P}^{2}$ amplitudes, have the opposite sign, so we see that there are actually two types of $\mathrm{O} p$-planes. These are denoted $\mathrm{O} p^{-}$and $\mathrm{O} p^{+}$for the $\mathrm{SO}(2 n)$ and $\mathrm{USp}(2 n)$ cases, respectively. Comparing the Möbius strip amplitude with the cylinder amplitude, one finds that the $\mathrm{RR}$ charge and tension of the $\mathrm{O} p$-planes (as compared with those of the $\mathrm{D} p$-branes) is given by $-2^{p-5}$ for $\mathrm{O}^{-}$, and $+2^{p-5}$ for $\mathrm{O} p^{+}$(hence the sign in the notation).

\subsection{Cohomology torsion variants}

Another way to see that there are (at least) two variants (at least for $p \leq 6$ ) is to note that the orientifold background admits a discrete degree of freedom corresponding to the holonomy of the $B$ field [i] $\left[\begin{array}{l}1 \\ 1\end{array}\right]$,

$$
b=\int_{\mathbb{R P}^{2}} \frac{B}{2 \pi}=0 \quad \text { or } \quad \frac{1}{2},
$$

where $\mathbb{R P}^{2} \subset \mathbb{R P}^{8-p}$ which surrounds the $\mathrm{O} p$-plane. A non-trivial holonomy contributes $e^{2 \pi i b}=-1$ in the $\mathbb{R P}^{2}$ amplitude, and therefore exchanges the $\mathrm{O} p^{-}$and $\mathrm{O} p^{+}$ planes. ${ }^{1}$ Since $B$ is odd under the projection, which maps opposite points in the

\footnotetext{
${ }^{1}$ It isn't obvious at this stage which variant should be identified with trivial holonomy, and which with non-trivial holonomy. This will be clarified in K-theory.
} 
double cover of $\mathbb{R P}^{8-p}$, the holonomy is an element of $H^{2}\left(\mathbb{R P}^{8-p}, \widetilde{\mathrm{U}(1)}\right)$, where $\widetilde{\mathrm{U}(1)}$ is the twisted $\mathrm{U}(1)$ bundle. The elements of $H^{2}\left(\mathbb{R P}^{8-p}, \widetilde{\mathrm{U}(1)}\right)$ are in turn classified topologically by the characteristic class

$$
[H]=[d B] \in H^{3}\left(\mathbb{R P}^{8-p}, \widetilde{\mathbb{Z}}\right)=\mathbb{Z}_{2},
$$

where $\widetilde{\mathbb{Z}}$ is the twisted $\mathbb{Z}$ bundle. The choice of torsion coincides with the holonomy $b$.

$\mathrm{RR}$ fields can also have a discrete holonomy, which leads to additional variants of the orientifold plane. For the $\mathrm{O} p$-plane with $p \leq 5$ the relevant holonomy is

$$
c=\int_{\mathbb{R P}^{5}-p} \frac{C_{5-p}}{2 \pi}=0 \quad \text { or } \quad \frac{1}{2}
$$

and is identified with a torsion $G_{6-p}$-field,

$$
\left[G_{6-p}\right] \in\left\{\begin{array}{ll}
H^{6-p}\left(\mathbb{R P}^{8-p}, \mathbb{Z}\right) & \text { for } p=0,2,4 \\
H^{6-p}\left(\mathbb{R P}^{8-p}, \widetilde{\mathbb{Z}}\right) & \text { for } p=1,3,5
\end{array}\right\}=\mathbb{Z}_{2} .
$$

The choice of a non-trivial $c$ is denoted $\widetilde{\mathrm{O} p}{ }^{ \pm}$. Unlike the previous case, the charges of these variants cannot be computed in perturbation theory, since they involve a RR background. Instead, one must appeal to arguments involving strong coupling. For example, the O3-plane has four variants corresponding to $H$ and $G_{3}$ torsion, both of which take values in $H^{3}\left(\mathbb{R P}^{5}, \widetilde{\mathbb{Z}}\right)=\mathbb{Z}_{2}$. S-duality in type-IIB string theory exchanges $H$ and $G_{3}$, so $\mathrm{O}^{-}$and $\widetilde{\mathrm{O}}^{+}$are invariant, and $\mathrm{O}^{+}$is interchanged with $\widetilde{\mathrm{O}}^{-}$[i]. If we include D3-branes, string S-duality becomes Olive-Montonen duality in the worldvolume $N=4$ supersymmetric gauge theory. The $\mathrm{SO}(2 n)$ theory corresponding to $\mathrm{O}^{-}$is invariant under this duality, but the $\mathrm{USp}(2 n)$ theory of $\mathrm{O}^{+}$is related to an $\mathrm{SO}(2 n+1)$ theory $[\underline{1} \overline{1} \overline{0}]$. We can therefore interpret the $\widetilde{\mathrm{O}} 3^{-}$plane as an $\mathrm{O}^{-}$plane with an additional fractional $(1 / 2)$ D3-brane. The fourth variant, $\widetilde{\mathrm{O}^{+}}+$, corresponds to turning on $H$ torsion on $\widetilde{\mathrm{O}}^{-}$. This exchanges the orthogonal gauge group with a symplectic one, $\mathrm{USp}(2 n)$. The theory is perturbatively the same as for $\mathrm{O}^{+}$, but the BPS dyon spectrum is different [2]. This $\mathrm{U} \operatorname{Sp}(2 n)$ theory is self-dual.

There are analogous strong coupling arguments for type-IIA O2 planes [i]

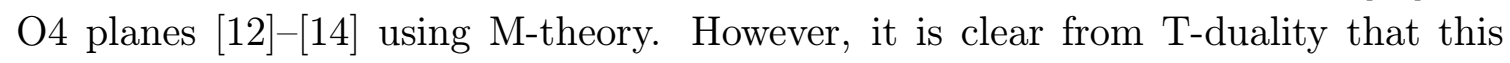
pattern persists for all $p \leq 5$ (see table $\left.\underline{\Omega}_{1}^{\overline{1}}\right)$.

The case $p=6$ is special, since $\left[G_{0}\right] \in H^{0}\left(\mathbb{R} \mathrm{P}^{8-p}, \mathbb{Z}\right)=\mathbb{Z}$, rather than $\mathbb{Z}_{2}$. These classes correspond to the allowed (integral) values of the cosmological constant in

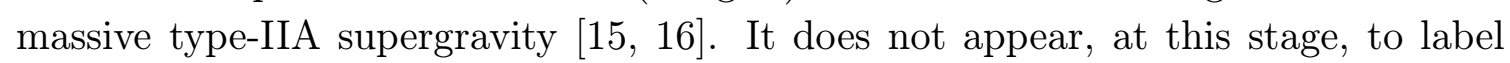
distinct O6-planes, but rather different IIA backgrounds in which an O6-plane can sit. One might therefore conclude that the O6-plane only has two variants, $\mathrm{O}^{-}$and $\mathrm{O}^{+}$. However, we shall see in section ${ }_{-1}^{5}$, that there is another variant, which has the properties (namely charge and tension) of the $\widetilde{\mathrm{Op}^{-}}$-planes, and is therefore similarly denoted $\widetilde{\mathrm{O}}^{-}$(see also [1] $\left.\left.\underline{1}_{1} \bar{q}_{1}\right]\right)$. 
For $p=0$ and 1 there is another possible holonomy given by

$$
c^{\prime}=\int_{\mathbb{R P}^{1-p}} \frac{C_{1-p}}{2 \pi}=0 \quad \text { or } \quad \frac{1}{2} .
$$

This is related to the torsion groups $H^{1}\left(\mathbb{R P}^{7}, \widetilde{\mathbb{Z}}\right)$ and $H^{2}\left(\mathbb{R P}^{8}, \mathbb{Z}\right)$. We denote the corresponding variants $\widehat{\mathrm{O} 1}$ and $\widehat{\mathrm{O} 0}$. When both $\mathrm{RR}$ torsions are turned on we get more variants, $\widehat{\widetilde{\mathrm{O} 1}}$ and $\widehat{\widetilde{\mathrm{O} 0}}$. The charges of these variants were not known. Using Ktheory, we will be able to determine their charges. We will also see that some of these variants are removed when one interprets $\mathrm{RR}$ fields in K-theory. For the O2-plane, one could also consider a $G_{0}$ background, which takes values in $H^{0}\left(\mathbb{R} \mathrm{P}^{6}, \mathbb{Z}\right)=\mathbb{Z}$. The situation is similar to the O6-plane case above, and will also be addressed in section

\subsection{Brane realization of torsion}

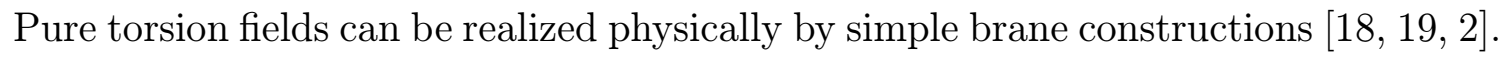
Consider an $\mathrm{O} p$-plane $(p \leq 5)$ situated along the directions $\left\{x^{1}, \ldots, x^{p-1}, x^{6}\right\}$, and either an NS5-brane along $\left\{x^{1}, \ldots, x^{5}\right\}$ for the case of the $H$ field, or a $\mathrm{D}(p+2)$-brane along $\left\{x^{1}, \ldots, x^{p-1}, x^{7}, x^{8}, x^{9}\right\}$ for the case of the $G_{6-p}$ field (figure $\mathbf{i}_{1}^{1}$ )..$^{2}$ In both cases the brane intersects the orientifold plane along $\left\{x^{1}, \ldots, x^{p-1}\right\}$, and therefore forms a "domain wall" on the Op-plane. The brane is "fractional" in the sense that it is its own image under the orientifold projection, and can therefore not move off the orientifold plane. It is therefore a source of $1 / 2$ unit of flux in the reduced space, i.e.

$$
\int_{\mathbb{S} 3,1} \frac{H}{2 \pi}=\frac{1}{2}
$$

for the NS5-brane, and

$$
\int_{\mathbb{S}^{6-p, 1}} \frac{G_{6-p}}{2 \pi}=\frac{1}{2}
$$

for the $\mathrm{D}(p+2)$-brane. ${ }^{3}$ Now remove two small disks at the intersection of the sphere with the orientifold plane, and apply Stoke's law to get

$$
\Delta \int_{\mathbb{R P}^{2}} \frac{B}{2 \pi}=\Delta \int_{\mathbb{R P}^{5-p}} \frac{C_{5-p}}{2 \pi}=\frac{1}{2}
$$

for the difference in the holonomy between the two sides. The field strengths $H$ and $G_{6-p}$ are trivial (as differential forms) at infinity, but the above result shows that there is relative torsion between the two pieces of the $\mathrm{O} p$-plane. The NS5-brane interchanges the $\mathrm{O}^{-}$and $\mathrm{O} p^{+}$planes, and the $\mathrm{D}(p+2)$-brane interchanges the $\mathrm{O} p$ and $\widetilde{\mathrm{O} p}$ planes.

\footnotetext{
${ }^{2} \mathrm{~A}$ similar construction can be made for the additional RR torsion $\left(c^{\prime}\right)$ in the case of $p=0,1$, using an intersection with a D6-brane and D7-brane, respectively.

${ }^{3}$ We use $\mathbb{S}^{l, m}$ to denote the sphere in the reduced space $\mathbb{R}^{l, m} \equiv \mathbb{R}^{l+m} / \mathbb{Z}_{2}$, where the $\mathbb{Z}_{2}$ inverts $l$ of the coordinates. In particular $\mathbb{S}^{l, 0} \sim \mathbb{R P}^{l-1}$.
} 


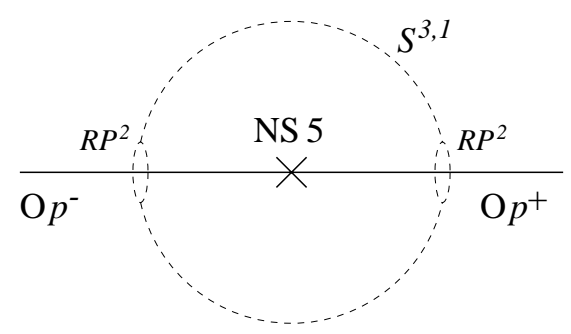

a

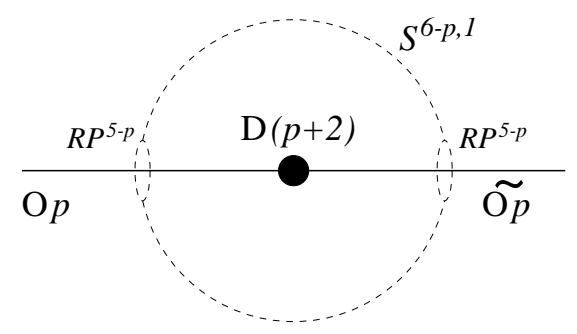

$\mathrm{b}$

Figure 1: Brane realization of $(a) H$ torsion, $(b) G_{6-p}$ torsion.

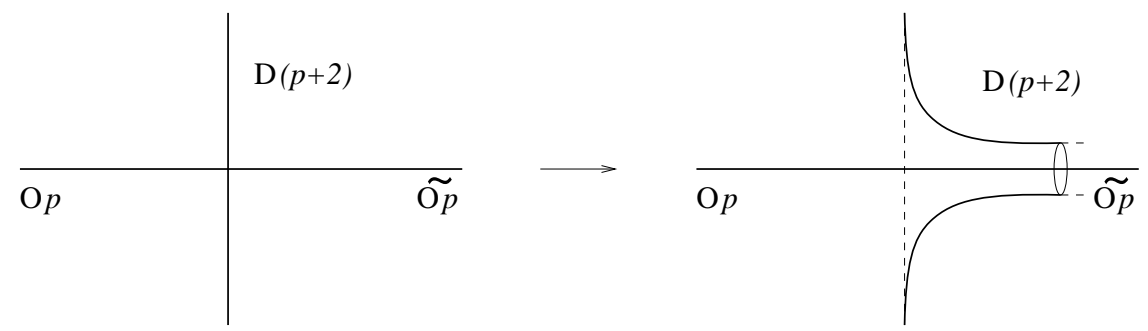

Figure 2: Deforming the intersecting brane to a wrapped brane.

For two coincident branes the flux is 1 , but they are now free to move off the orientifold plane (in opposite directions). The torsion part of the field is therefore trivial, and the orientifold plane remains unchanged.

For $p=6$ the D-brane picture (figure $i_{-1}^{1} b$ ) is special, in that $G_{0}$ is created by a D8-brane, which fills the entire transverse space. The D8-branes can therefore never move off the orientifold plane, and this is consistent with the fact that $G_{0}$ is integral rather than torsion.

We can try to use this picture to understand the relative charges of the $\widetilde{\mathrm{O} p}{ }^{ \pm}$ planes. Consider a "deformation" of the intersecting D-brane configuration, in which the $\mathrm{D}(p+2)$-brane wraps $\mathbb{R}^{p} \times \mathbb{R P}^{2}$ at $x^{6} \rightarrow \infty$, and "opens up" into a flat $\mathbb{R}^{p+2}$ at $x^{6}=0$ (figure $\bar{z}$ ). The $\mathbb{R P}^{2}$ is not a homology cycle in the bulk, so the wrapped part of the brane shrinks to zero size, giving the original intersection configuration. If we assume that the world-volume gauge field on the $\mathrm{D}(p+2)$-brane satisfies $\left[2 \overline{2} \bar{O}_{1}, \overline{1}_{1} \bar{I}_{1}\right]$

$$
\int_{\mathbb{R P}^{2}} \frac{F}{2 \pi}=\frac{1}{2} \quad \bmod \mathbb{Z}
$$

then the charge of the $\mathrm{O} p^{-}$plane would shift by $1 / 2$, due to the coupling $\int F \wedge C_{p+1}$. On the other hand the charge of $\mathrm{O}^{+}$could only change by an integral amount due the additional coupling $\int B \wedge C_{p+1}$, since the $B$ field also has $1 / 2$ unit of flux on $\mathbb{R P}^{2}$. This would therefore explain the observed relative charges of these two variants. A result similar to $\left(\overline{2} . \overline{1} 2^{\prime}\right)$ was obtained in [2] non-trivial. However $\mathbb{R P}^{2}$ is non-orientable, so the question remains open. 


\section{Orientifolds in K-theory}

In ordinary (non-orientifold) type-IIA and -IIB backgrounds RR fluxes take values in $K(X)$ and $K^{-1}(X)$, respectively [i] . This complements the fact that $\mathrm{RR}$ charges, or D-branes, are classified by $K^{-1}(X)$ and $K(X)$, respectively. ${ }^{4}$ One way to think about this, which will be useful in generalizing to orientifolds, is to divide the space into two regions using a domain wall made of D8-branes and anti-D8-branes in IIA, and non-BPS D8-branes in IIB. Differences in the values of the RR fluxes between the two regions are then in one-to-one correspondence with the $\mathrm{RR}$ charges carried by the D8-brane system, and are therefore classified by $K(X)$ in IIA, and by $K^{-1}(X)$ in IIB. The groups are exchanged relative to the D-brane classification since there we start with a D9- $\overline{\mathrm{D} 9}$ system in IIB, and non-BPS D9-branes in IIA.

\subsection{Real K-theory}

The orientifold backgrounds of interest are defined by a $\mathbb{Z}_{2}$ projection on closed strings that includes world-sheet parity $\Omega$, and an action on the open string ChanPaton factors that squares to either 1 or -1 . In K-theoretic terms, one is given an involution $\tau$ on the space $X$, and an antilinear involution on the bundles that commutes with $\tau$. This defines the real and quaternionic K-theory groups $K R(X)$ and $K H(X)$, depending on whether the involution on the bundles squares to 1 or $-1[\overline{2} \overline{2} \overline{2}]$. Higher groups can then be defined by (assuming compact support)

$$
K R^{p, q}(X)=K R\left(X \times \mathbb{R}^{p, q}\right)
$$

where the involution acts on $p$ coordinates in $\mathbb{R}^{p, q}$, and similarly for $K H$. These satisfy the periodicity conditions

$$
\begin{aligned}
& K R^{p, q}(X)=K R^{p+1, q+1}(X) \\
& K R^{p, q}(X)=K R^{p+8, q}(X)
\end{aligned}
$$

and similarly for $K H$. The first implies that $K R^{p, q}$ depends only on the difference $p-q$, so we denote $K R^{p, q}(X)=K R^{p-q}(X)$. In addition $K R$ and $K H$ are related by

$$
K R^{-n}(X)=K H^{-n+4}(X)
$$

Another variant of real K-theory can be defined by considering an involution that also exchanges the two bundles $E$ and $F$. Physically this corresponds to the inclusion of the operator $(-1)^{F_{L}}$ (e.g. for $p=3 \bmod 4$ in $\left({ }_{2} 2 . \overline{1}_{1}^{\prime}\right)$ ), which exchanges the branes and antibranes. The corresponding groups are denoted $K R_{ \pm}(X)$ and $K H_{ \pm}(X)$, and

\footnotetext{
${ }^{4}$ If $X$ is non-compact, then D-branes are actually classified by K-theory with compact support, $K_{\text {cpct }}^{-1}(X)$ and $K_{\text {cpct }}(X)$, or more precisely by the kernel of the natural maps from these groups to the ones without compact support.
} 


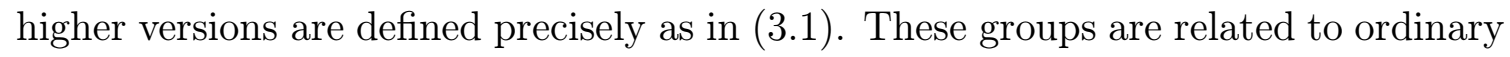
real K-theory as follows,

$$
K R_{ \pm}(X)=K R\left(X \times \mathbb{R}^{0,2}\right)=K R^{-2}(X)
$$

and similarly for $K H_{ \pm} \cdot{ }^{5}$

\subsection{RR fields in orientifold backgrounds}

To classify RR fields in the background of an orientifold $p$-plane we divide the transverse space $\mathbb{R}^{9-p}$ by wrapping D8-branes on the transverse sphere $\mathbb{S}^{8-p}$. In IIA we again consider both D8-branes and anti-D8-branes, and in IIB we use the non-BPS D8-branes. The orientifold projection (2... $\left.\overline{1}_{1}\right)$ acts freely on the sphere, giving $\mathbb{R P}^{8-p}$, and the corresponding K-groups are obtained by keeping track of how the projection acts on the D8-branes and their bundles.

For example, the O0 projection $\mathbb{R}^{9} / \mathcal{I}_{9} \Omega$ in IIA maps a D8-brane wrapping $\mathbb{S}^{8}$ with a given orientation to a D8-brane wrapping it with the opposite orientation. It therefore exchanges wrapped D8-branes with wrapped anti-D8-branes, and viceversa. The group is therefore either $K R_{ \pm}\left(\mathbb{S}^{9,0}\right)$ or $K H_{ \pm}\left(\mathbb{S}^{9,0}\right)$, depending on whether the action on the Chan-Paton bundle squares to 1 or -1 . Following the general arguments of [9. we associate the former to the $\mathrm{O}^{-}$-plane, and the latter to the $\mathrm{O}^{+}$-plane.

The usual 8-fold periodicity of orientifolds (and real K-theory) implies that for the $\mathrm{O}^{-}$and $\mathrm{O}^{+}$planes the groups are also $K R_{ \pm}$and $K H_{ \pm}$, respectively. This is somewhat counterintuitive, in that a D8-brane gets mapped to an image D8-brane and not an anti-D8-brane. However recall that the D8-branes wrap the transverse sphere, which in this case is just two points on either side of the O8-plane. This means that the orientation of the "wrapped" D8-brane is reversed on the other side. The projection maps the D8-brane to a D8-brane of the same orientation on the other side, so it looks like a "wrapped" anti-D8-brane.

The appropriate groups for the other orientifold planes can be similarly determined. The results are summarized in table 2

Using (13.2. $K H^{p-10}\left(\mathbb{S}^{9-p, 0}\right)=K R^{p-6}\left(\mathbb{S}^{9-p, 0}\right)$ for $\mathrm{O} p^{+}$. Note that, as expected, the groups which classify RR fields are shifted by one relative to the groups which classify RR charges,

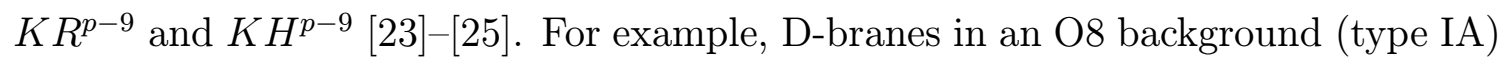

\footnotetext{
${ }^{5}$ This relation is the analog in real K-theory of Hopkins' relation in complex equivariant K-theory, $K_{ \pm}(X)=K_{\mathbb{Z}_{2}}\left(X \times \mathbb{R}^{1,1}\right)$. The latter can be understood as the failure of ordinary Bott periodicity when one tries to construct the Dirac index map: $K_{\mathbb{Z}_{2}}\left(X \times \mathbb{R}^{1,1}\right) \rightarrow K_{\mathbb{Z}_{2}}(X)$. Orientation reversal on the $\mathbb{R}^{2}$ exchanges the two spinor chiralities, and therefore gives $K_{ \pm}(X)$ rather than $K_{\mathbb{Z}_{2}}(X)$. In the real case it's exactly the opposite, since complex conjugation also exchanges the two chiralities. Bott periodicity works for $\mathbb{R}^{1,1}(3.21)$, and fails for $\mathbb{R}^{0,2}$ and $\mathbb{R}^{2,0}$. The former gives $K R_{ \pm}(X)$, and the latter $K H_{ \pm}(X)$.
} 


\begin{tabular}{|l|l|}
\hline$O p^{-}$ & K-group \\
\hline \hline$O 0^{-}$ & $K R_{ \pm}\left(S^{9,0}\right)=\mathbb{Z} \oplus \mathbb{Z}_{2}$ \\
$O 1^{-}$ & $K R^{-1}\left(S^{8,0}\right)=\mathbb{Z} \oplus \mathbb{Z}_{2}$ \\
$O 2^{-}$ & $K R\left(S^{7,0}\right)=\mathbb{Z} \oplus \mathbb{Z}$ \\
$O 3^{-}$ & $K H_{ \pm}^{-1}\left(S^{6,0}\right)=\mathbb{Z}$ \\
$O 4^{-}$ & $K H_{ \pm}\left(S^{5,0}\right)=\mathbb{Z}$ \\
$O 5^{-}$ & $K H^{-1}\left(S^{4,0}\right)=\mathbb{Z}$ \\
$O 6^{-}$ & $K H\left(S^{3,0}\right)=\mathbb{Z} \oplus \mathbb{Z}$ \\
$O 7^{-}$ & $K R_{ \pm}^{-1}\left(S^{2,0}\right)=\mathbb{Z}$ \\
O8 & $K R_{ \pm}\left(S^{1,0}\right)=\mathbb{Z}$ \\
\hline
\end{tabular}

\begin{tabular}{|l|l|}
\hline$O p^{+}$ & K-group \\
\hline \hline$O 0^{+}$ & $K H_{ \pm}\left(S^{9,0}\right)=\mathbb{Z}$ \\
$O 1^{+}$ & $K H^{-1}\left(S^{8,0}\right)=\mathbb{Z}$ \\
$O 2^{+}$ & $K H\left(S^{7,0}\right)=\mathbb{Z} \oplus \mathbb{Z}$ \\
$O 3^{+}$ & $K R_{ \pm}^{-1}\left(S^{6,0}\right)=\mathbb{Z}$ \\
$O 4^{+}$ & $K R_{ \pm}\left(S^{5,0}\right)=\mathbb{Z} \oplus \mathbb{Z}_{2}$ \\
$O 5^{+}$ & $K R^{-1}\left(S^{4,0}\right)=\mathbb{Z} \oplus \mathbb{Z}_{2}$ \\
$O 6^{+}$ & $K R\left(S^{3,0}\right)=\mathbb{Z} \oplus \mathbb{Z}$ \\
$O 7^{+}$ & $K H_{ \pm}^{-1}\left(S^{2,0}\right)=\mathbb{Z}$ \\
$O 8^{+}$ & $K H_{ \pm}\left(S^{1,0}\right)=\mathbb{Z}$ \\
\hline
\end{tabular}

Table 2: Orientifold K-theory groups for RR fields.

are classified by $K R^{-1}$, whereas $\mathrm{RR}$ fields take values in $K R_{ \pm}=K R^{-2}$. To compute the groups we used the following decomposition isomorphism due to Atiyah [i2 $\overline{2}]$

$$
K R^{-n}\left(\mathbb{S}^{m, 0}\right)=K R^{m-n+1}(p t .) \oplus K R^{-n}(p t .) \quad(m \geq 3),
$$

and our knowlege of the real K-groups of a point,

$$
K R^{-m}(p t .)=\left\{\mathbb{Z}, \mathbb{Z}_{2}, \mathbb{Z}_{2}, 0, \mathbb{Z}, 0,0,0\right\} \bmod 8 .
$$

\subsection{Comparing with cohomology}

The above results agree with cohomology in some cases, and differ in other cases. The free $(\mathbb{Z})$ part of K-theory always agrees with cohomology. This corresponds to the RR flux of the orientifold $p$-plane itself, i.e. $G_{8-p}$. For $p=2,6$ there is an additional free part corresponding to $G_{0}$, which also agrees with cohomology. The difference between K-theory and cohomology can only be in the torsion subgroup. ${ }^{6}$

For example, the relevant cohomologies for orientifold 5-planes are

$$
H^{3}\left(\mathbb{R} P^{3}, \mathbb{Z}\right) \oplus H^{1}\left(\mathbb{R} P^{3}, \widetilde{\mathbb{Z}}\right)=\mathbb{Z} \oplus \mathbb{Z}_{2}
$$

The first term corresponds to the integral $G_{3}$ flux of the O5-plane, and the second is $G_{1}$ torsion, which gives the $\widetilde{\mathrm{O} 5}$ variants. Let us once again stress that these cohomologies do not incorporate the fractional shift in the $G_{3}$ flux of $\widetilde{\mathrm{O}}^{-}$relative to $\mathrm{O} 5^{-}$. In K-theory we find

$$
\begin{aligned}
K H^{-1}\left(\mathbb{S}^{4,0}\right) & =\mathbb{Z} & & \text { for } \mathrm{O5}^{-} \\
K R^{-1}\left(\mathbb{S}^{4,0}\right) & =\mathbb{Z} \oplus \mathbb{Z}_{2} & & \text { for } \mathrm{O5}^{+} .
\end{aligned}
$$

How should we interpret the absence of the torsion subgroup in the first case? Clearly we need to understand better how to relate (or "lift") cohomology to K-theory.

\footnotetext{
${ }^{6}$ This is a consequence of the Chern isomorphism, e.g. $K(X) \times \mathbb{Q} \simeq H^{\text {even }}(X, \mathbb{Q})$. In the real case the cohomologies will be twisted or normal, according to the action of the orientifold on the corresponding RR field $(\underline{2} .2 \overline{2})$.
} 
There is in fact a systematic algorithm which does exactly this, known as the Atiyah-Hirzebruch spectral sequence (AHSS) $\left[\overline{2}_{-1} \overline{6}_{i}{ }^{7}{ }^{7}\right.$ The basic idea of the AHSS is to compute $K(X)$ using a sequence of successive approximations, starting with integral cohomology $H^{*}(X, \mathbb{Z})$. Each successive step is given by the cohomology of a differential $d_{r}$ that acts on classes in the previous step. Thus classes which are not $d_{r}$-closed are removed in the new approximation, and classes which are $d_{r}$-exact are trivial. For example, $d_{1}$ is just the usual co-boundary operator acting on co-chains (which are the zero'th step), so the first step is ordinary de-Rahm cohomology. Higher differentials, if they are non-trivial, will refine the approximation by removing some cohomology classes, and trivializing others. The first non-trivial higher differential

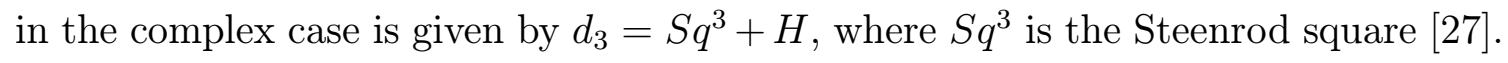
The only other differential which may be non-trivial in ten dimensions is $d_{5}$.

The AHSS can be extended to real K-theory as well. In the appendix we describe how to do this in the case of a freely acting involution, such as the ones appearing in table $\underset{i}{-i}$ In this case the AHSS follows a similar pattern to the complex case, with the modification that the cohomologies appearing in the first approximation can be twisted or normal, in accordance with the orientifold action on the RR

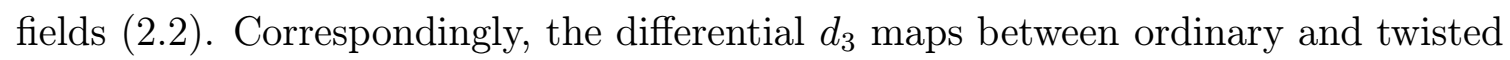
cohomologies, and must therefore itself be twisted. The precise form of $d_{3}$ is not known, but it can presumably be expressed in the form $d_{3}=\widetilde{S q^{3}}+H$, where $\widetilde{S q^{3}}$ is a twisted version of the Steenrod square. Here the second term is torsion, since $[H] \in H^{3}\left(\mathbb{R} P^{8-p}, \widetilde{\mathbb{Z}}\right)=\mathbb{Z}_{2}$. As we have seen before, this provides the difference between the $\mathrm{O} p^{-}$-plane and the $\mathrm{O} p^{+}$-plane. We shall assume that the first term is trivial in both cases, and justify this assumption in hindsight. We shall also see that $d_{5}$ (which is untwisted) is trivial in all cases.

Actually, what one really computes in the AHSS is an "associated graded complex" of $K(X)$ (or any of its variants), denoted $\operatorname{Gr} K(X)$. This is defined in terms of a filtration of $K(X), K_{n}(X) \subseteq K_{n-1}(X) \subseteq \cdots \subseteq K_{0}(X)=K(X)$, as

$$
\operatorname{Gr} K(X)=\oplus_{p} K_{p}(X) / K_{p+1}(X),
$$

where $K_{p}(X)$ is the subgroup of classes in $K(X)$ which are trivial on the $(p-1)$ tree of $X$. The successive ratios appearing in $\left({ }_{2} .10^{\prime}\right)$ are precisely the objects one computes by the AHSS. In particular, in the first order approximation

$$
K_{p}(X) / K_{p+1}(X)= \begin{cases}H^{p}(X, \mathbb{Z}) & \text { for } p \text { even } \\ 0 & \text { for } p \text { odd }\end{cases}
$$

To get the actual subgroups $K_{p}(X)$, and in particular $K(X)$ itself, requires solving extension problems of the form

$$
0 \rightarrow K_{p+1}(X) \rightarrow K_{p}(X) \rightarrow K_{p}(X) / K_{p+1}(X) \rightarrow 0 .
$$

\footnotetext{
${ }^{7}$ Introductory reviews of the AHSS for complex K-theory can be found in the appendix, as well

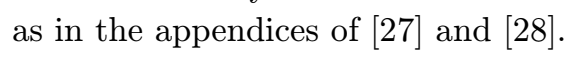


If the above exact sequence splits, i.e. if it is exact both ways, the extension is said to be trivial, and $K_{p}(X)=K_{p+1}(X) \oplus K_{p}(X) / K_{p+1}(X)$. If all the extensions are trivial we deduce recursively that $K(X)=\operatorname{Gr} K(X)$. In other words, K-theory is isomorphic to the ring of cohomologies that survive the higher differentials of the AHSS. On the other hand, if some of the extensions are non-trivial $K(X)$ will differ from its graded complex. In particular, the additive structure in K-theory will be different from that of the cohomology classes. Physically this means that RR fields of different degree become correlated.

Consider for example the space $X=\mathbb{R P}^{5}$, for which

$$
\begin{aligned}
\operatorname{Gr} K\left(\mathbb{R} P^{5}\right) & =H^{0}\left(\mathbb{R} P^{5}\right) \oplus H^{2}\left(\mathbb{R P}^{5}\right) \oplus H^{4}\left(\mathbb{R} P^{5}\right) \\
& =\mathbb{Z} \oplus \mathbb{Z}_{2} \oplus \mathbb{Z}_{2} .
\end{aligned}
$$

The relevant extension is given by

$$
\begin{aligned}
& 0 \rightarrow K_{3}\left(\mathbb{R P}^{5}\right) \rightarrow K_{2}\left(\mathbb{R P}^{5}\right) \rightarrow K_{2}\left(\mathbb{R P}^{5}\right) / K_{3}\left(\mathbb{R P} \mathrm{P}^{5}\right) \rightarrow 0 \\
& \|\quad\| \quad \| \\
& H^{4}=\mathbb{Z}_{2} \quad K_{1}\left(\mathbb{R P}^{5}\right) \quad H^{2}=\mathbb{Z}_{2}
\end{aligned}
$$

On the other hand it is known (see for example the appendix of [208]) that

$$
K_{1}\left(\mathbb{R} P^{5}\right)=\widetilde{K}\left(\mathbb{R P}^{5}\right)=\mathbb{Z}_{4}
$$

so this extension is non-trivial. The $\mathrm{RR} 2$-torsion and 4-torsion appearing in $H^{2}\left(\mathbb{R P}^{5}\right)$ and $H^{4}\left(\mathbb{R P}^{5}\right)$ become correlated in K-theory; twice the generator of the former corresponds to the generator of the latter.

In practical terms, one often needs additional information to determine whether an extension is trivial or not, which precludes the AHSS as a useful method for computing $K(X)$ (as opposed to $\operatorname{Gr} K(X)$ ). However it will be extremely useful in our case, since we already know the precise groups (table 2 able to determine all the extensions ( K-theory data with cohomology.

\section{Applications}

Having elaborated the K-theory machinery needed to classify RR fields surrounding orientifolds, we would now like to demonstrate three important implications of the fact that that RR fields are valued in K-theory rather than cohomology.

\subsection{Charge quantization}

As was previously stated, there are two separate issues related to the question of proper RR charge quantization. The first has to do with the absolute fractional 
charges (of the $\mathrm{O} p$-planes with $p \leq 4$, for example), and the second with the relative charge induced by $\mathrm{RR}$ torsion $\left(1 / 2\right.$ for $\mathrm{O}^{-}, 0$ for $\left.\mathrm{O}^{+}\right)$. The first issue encompasses the second, and should in principle be resolved by an analog of the relation [i]

$$
\frac{G(x)}{2 \pi}=\sqrt{\widehat{A}} \operatorname{ch}(x), \quad x \in K(X)
$$

for real K-theory. Unfortunately the generalization is not known, so we will concentrate on the issue of the relative charge. The main idea, as alluded to in the previous section, is that in K-theory RR fields of different degree become correlated via non-trivial extensions.

Note first that for the $\mathrm{O} 8$ and O7-planes K-theory is in complete agreement with cohomology. There is only a single RR flux, and therefore no possibility for a non-trivial extension. There are only two variants of these planes, $\mathrm{O} p^{-}$and $\mathrm{O} p^{+}$. In particular the O8-plane (O7-plane) cannot support a fractional D8-brane (D7brane). This is consistent with other arguments which rule out the possibility of a

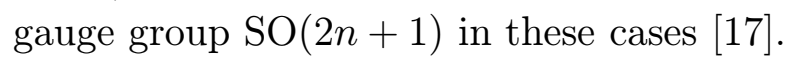

The O6-plane background supports two RR fluxes, $G_{2}$ and $G_{0}$, but both are integral. Since the differential $d_{3}$ (and $d_{5}$ ) is obviously trivial in this case, the AHSS terminates at the first approximation, i.e.

$$
\begin{aligned}
K_{0} / K_{1} & =H^{0}\left(\mathbb{R P}^{2}, \mathbb{Z}\right)=\mathbb{Z} \\
K_{1} / K_{2} & =0 \\
K_{2} & =H^{2}\left(\mathbb{R P} P^{2}, \widetilde{\mathbb{Z}}\right)=\mathbb{Z} .
\end{aligned}
$$

Using $K_{0}=K H\left(\mathbb{S}^{3,0}\right)$ and $K R\left(\mathbb{S}^{3,0}\right)$ for $\mathrm{O}^{-}$and $\mathrm{O}^{+}$, respectively, we can determine the filter groups uniquely,

$$
\begin{aligned}
& K_{0}=\mathbb{Z} \oplus \mathbb{Z} \\
& K_{1}=K_{2}=\mathbb{Z} .
\end{aligned}
$$

However the extension given by

$$
\begin{aligned}
& \begin{array}{c}
0 \rightarrow \quad K_{1} \\
\|
\end{array} \rightarrow \underset{\|}{ } \rightarrow \underset{\|}{K_{0}} \rightarrow \underset{K_{0} / K_{1}}{\|} \rightarrow 0 \\
& H^{2}=\mathbb{Z} \quad \mathbb{Z} \oplus \mathbb{Z} \quad H^{0}=\mathbb{Z}
\end{aligned}
$$

does not have a unique solution, so one cannot determine in this way whether and how the two RR fluxes are correlated. We will return to the O6-plane in section

The first case where this procedure gives a non-trivial result is the O5-plane. The cohomologies and K-theory for the O5-planes were given in (‥3. only possible non-triviality of $d_{3}$ could be in the map

$$
H^{0}\left(\mathbb{R} P^{3}, \widetilde{\mathbb{Z}}\right) \stackrel{d_{3}}{\longrightarrow} H^{3}\left(\mathbb{R} P^{3}, \mathbb{Z}\right) .
$$


However $H^{0}\left(\mathbb{R P} P^{3}, \widetilde{\mathbb{Z}}\right)=0$, so $d_{3}\left(\right.$ and $\left.d_{5}\right)$ is trivial for both $\mathrm{O}^{-}$and $\mathrm{O}^{+}$. The AHSS again terminates at cohomology, and we find

$$
\begin{aligned}
& K_{0}=K_{1}= \begin{cases}K H^{-1}\left(\mathbb{S}^{4,0}\right)=\mathbb{Z} & \text { for } \mathrm{O}^{-} \\
K R^{-1}\left(\mathbb{S}^{4,0}\right)=\mathbb{Z} \oplus \mathbb{Z}_{2} & \text { for } \mathrm{O}^{+}\end{cases} \\
& K_{2}=K_{3}=H^{3}\left(\mathbb{R P}^{3}, \mathbb{Z}\right)=\mathbb{Z} \quad \text { for both }
\end{aligned}
$$

This time we find that the extension given by

$$
\begin{aligned}
& \begin{array}{l}
K_{2} \\
\|
\end{array} \rightarrow K_{1} \rightarrow \underset{\|}{K_{1} / K_{2}} \rightarrow 0 \\
& H^{3}=\mathbb{Z} \quad H^{1}=\mathbb{Z}_{2}
\end{aligned}
$$

is trivial for $\mathrm{O}^{+}$, but non-trivial for $\mathrm{O}^{-}$. The generator of $\mathrm{H}^{3}$ maps to the generator of (the free part of) $K_{1}$ for $\mathrm{O}^{+}$, but to twice the generator for $\mathrm{O}^{-}$. In the latter case the generator of $K_{1}$ maps to non-trivial torsion in $H^{1}$. We therefore see that $G_{1}$ torsion produces a half-integral shift in the flux of $G_{3}$ for the $\mathrm{O}^{-}$plane, and has no effect on the charge of the $\mathrm{O}^{+}$plane.

The story repeats along similar lines for the lower $\mathrm{O}^{-}$planes. The relevant cohomologies are $H^{8-p}\left(\mathbb{R P}^{8-p}\right)=\mathbb{Z}$ and $H^{6-p}\left(\mathbb{R P}^{8-p}\right)=\mathbb{Z}_{2}$, which are appropriately twisted or untwisted. The differential $d_{3}$ is trivial since $H=0$ (and we assume $\widetilde{S q^{3}}=0$ ). Possible non-trivialities of $d_{5}$ could arise from the (twist preserving) maps

$$
\begin{aligned}
& H^{3-p}\left(\mathbb{R} \mathrm{P}^{8-p}\right) \longrightarrow H^{8-p}\left(\mathbb{R P}^{8-p}\right) \\
& H^{1-p}\left(\mathbb{R} \mathrm{P}^{8-p}\right) \longrightarrow H^{6-p}\left(\mathbb{R P}^{8-p}\right) \\
& H^{6-p}\left(\mathbb{R} \mathrm{P}^{8-p}\right) \longrightarrow H^{11-p}\left(\mathbb{R P}^{8-p}\right),
\end{aligned}
$$

however in all cases the maps are found to be trivial. The relevant extension is

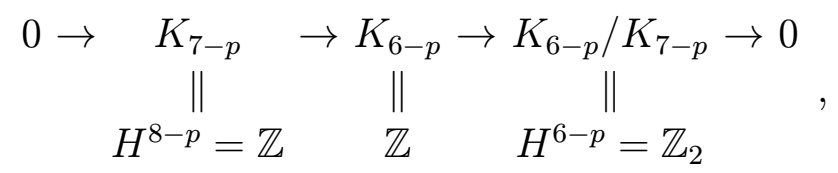

so the half-integral part of the $G_{8-p}$ flux, i.e. the charge of the orientifold plane, is determined by the $G_{6-p}$ torsion.

Actually, for $p \leq 2$ there is an additional RR cohomology $H^{2-p}$, i.e.

$$
\begin{array}{lc}
H^{0}\left(\mathbb{R P} P^{6}, \mathbb{Z}\right)=\mathbb{Z} & \text { for } p=2 \\
H^{1}\left(\mathbb{R P} P^{7}, \widetilde{\mathbb{Z}}\right)=\mathbb{Z}_{2} & \text { for } p=1 \\
H^{2}\left(\mathbb{R P} P^{8}, \mathbb{Z}\right)=\mathbb{Z}_{2} & \text { for } p=0 .
\end{array}
$$

As with the other cohomologies, these lift unobstructed to the graded complex; $d_{3}$ is trivial since $H=0$ for $\mathrm{O} p^{-}$planes, and $d_{5}$ is trivial since $H^{7-p}\left(\mathbb{R P}^{8-p}\right.$ ) (appropriately 
normal or twisted) is trivial. There is also an additional extension to solve,

$$
0 \rightarrow K_{3-p} \rightarrow K_{2-p} \rightarrow \underset{K_{2-p} / K_{3-p}}{K^{2-p}} \rightarrow 0
$$

One can show that in all three cases $K_{3-p}=\mathbb{Z}$, and $K_{2-p}=K_{0}$, which is $\mathbb{Z} \oplus \mathbb{Z}$ for $p=2$, and $\mathbb{Z} \oplus \mathbb{Z}_{2}$ for $p=0,1$. The $\mathrm{O}^{-}$case is similar to the $\mathrm{O}^{-}$case above; there is no unique solution to the extension, so one must use a different method to determine the effect of $G_{0}$. We will return to this problem in section 5. For $\mathrm{O}^{-}$ and $\mathrm{O}^{-}$the extension is trivial, so $G_{2-p}$ torsion does not shift their charge. ${ }^{8}$ The variants we called $\widehat{\mathrm{O}}^{-}$and $\widehat{\mathrm{O}}^{-}$therefore have the same charge (and tension) as the original torsion-free planes. It would be interesting to investigate whether (and how) the gauge theories on D1-branes and D0-branes in the presence of these exotic orientifold variants differ from the ordinary $\mathrm{SO}(2 n)$ theories on $\mathrm{O}^{-}$and $\mathrm{O}^{-}$.

The story becomes even more interesting for the lower $\mathrm{O} p^{+}$planes, as we will see next.

\subsection{Equivalent orientifolds}

$\mathrm{O} p^{+}$planes correspond to $\mathrm{O} p^{-}$planes with non-trivial $H$-torsion. This will modify the AHSS for $p \leq 3$ through the differential $d_{3}$. In particular, for the $\mathrm{O}^{+}$plane

$$
d_{3}: H^{0}\left(\mathbb{R} P^{5}, \mathbb{Z}\right) \stackrel{\cup H}{\longrightarrow} H^{3}\left(\mathbb{R} P^{5}, \widetilde{\mathbb{Z}}\right)
$$

is a surjective map from $\mathbb{Z}$ to $\mathbb{Z}_{2}$. $G_{3}$ torsion therefore lifts to a trivial class, and the graded complex is simply $\operatorname{Gr} K R_{ \pm}^{-1}\left(\mathbb{S}^{6,0}\right)=\mathbb{Z}$. As with the $\mathrm{O} 5^{+}$plane, the extensions are trivial, so the charge doesn't shift. However, the new feature here is that, since the two $G_{3}$ fluxes are equivalent in K-theory, the two variants $\mathrm{O}^{+}$and $\widetilde{\mathrm{O}}^{+}$are equivalent as well. Physically this can be understood as follows. Equation ( implies the existence of a constant 0 -form $\omega_{0}$, such that

$$
G_{3}=H \cup \omega_{0}
$$

and the two $\mathrm{O}^{+}$variants are related by a shift $\omega_{0} \rightarrow \omega_{0}+1$. On the other hand, the NSNS and RR 3-torsions of the two variants are related by an $\operatorname{SL}(2, \mathbb{Z})$ transformation

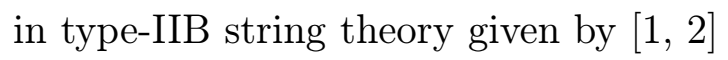

$$
T=\left[\begin{array}{ll}
1 & 1 \\
0 & 1
\end{array}\right]
$$

which also shifts the axion $C_{0} \rightarrow C_{0}+1$. We therefore identify $\omega_{0}$ with $C_{0}$.

\footnotetext{
${ }^{8}$ This is also consistent with the fact that the branes which create this torsion, i.e. a D6-brane for $\mathrm{O}^{-}$and a D7-brane for $\mathrm{O}^{-}$, cannot support the end of a D-string.
} 
Note that this transformation is global; in a background with several $\mathrm{O}^{+}$planes, it changes all of them into $\widetilde{\mathrm{O}^{+}}$planes. In particular, a configuration with two $\mathrm{O}^{+}$ planes at opposite points on a circle is not equivalent to a configuration with one $\mathrm{O}^{+}$and one $\widetilde{\mathrm{O}^{+}}{ }^{+}$. This is also consistent with the fact that these configurations are

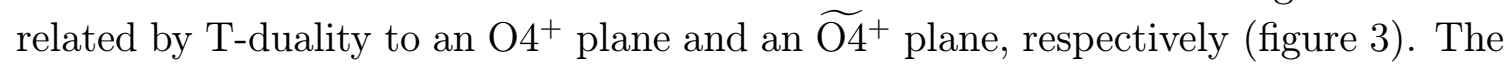
latter are inequivalent, since they differ by a $\left(G_{2}\right)$ torsion class which is not trivial in K-theory.

For $p \leq 2$ we similarly find that $G_{6-p}$ torsion can be written as

$$
G_{6-p}=H \cup \omega_{3-p},
$$

where $\omega_{3-p} \in H^{3-p}\left(\mathbb{R P}^{8-p}\right)=\mathbb{Z}_{2}$ (appropriately normal or twisted), so $\mathrm{O} p^{+}$and $\widetilde{\mathrm{O} p}+$ are equivalent in K-theory. For $p=2$ there is again an interesting physical picture. Consider the lift of $\mathrm{O}^{+}$to M-theory ${ }_{1}^{1} 1,1,2,1$. This corresponds to an $\mathrm{OM} 2^{+}$ plane and and $\mathrm{OM} 2^{-}$plane located at opposite points on the circle. ${ }^{9}$ The $\widetilde{\mathrm{O}}{ }^{+}$plane is then simply gotten by exchanging the positions of the two OM2 planes, which is equivalent to the shift $x^{10} \rightarrow x^{10} \pm \pi$. On the other hand, the M-theory shift $x^{10} \rightarrow x^{10}+\xi(-\pi \leq \xi \leq \pi)$ in flat space reduces to a $\mathrm{U}(1)$ gauge transformation in type-IIA string theory,

$$
\delta C_{1}=d \xi, \delta C_{3}=\frac{B}{2 \pi} \wedge d \xi
$$

In the OM2 background we require $\xi\left(-x^{i}\right)=-\xi\left(x^{i}\right)$, where $x^{i}(i=3, \ldots, 9)$ are the coordinates transverse to the $\mathrm{O} 2^{+}$-plane. In particular, at the location of the orientifold plane $\xi(0)=0$ or $\pi(\bmod 2 \pi)$ only, and this labels the discrete choice of the $\mathrm{O}^{+}$variant. Comparing ( $\left(\bar{A} . \overline{1} \overline{6}_{1}^{\prime}\right)$ with $\left(\bar{A}_{-} \overline{1} \overline{1} \overline{5}_{1}^{\prime}\right)$ we are led to identify $\omega_{1}$ with flat RR 1-forms $C_{1} \in H^{1}\left(\mathbb{R P}^{6}, \widetilde{\mathbb{Z}}\right)=\mathbb{Z}_{2}$.

In this case there are two possible non-trivial transformations for a pair of $\mathrm{O}_{2}^{+}$ planes at opposite points on a circle,

$$
\xi_{1}\left(x^{9}\right)=\left\{\begin{array}{rlr}
-\pi & \text { for } & -\pi<x^{9}<0 \\
\pi & \text { for } \quad 0<x^{9}<\pi
\end{array}, \quad \xi_{2}\left(x^{9}\right)=x^{9} .\right.
$$

In the first case both are transformed into $\widetilde{\mathrm{O} 2}{ }^{+}$planes, but in the second case only one $\left(\right.$ at $\left.x^{9}=\pi\right)$ is transformed. The configuration with one $\mathrm{O}_{2}^{+}$and one $\widetilde{\mathrm{O}_{2}}{ }^{+}$is therefore equivalent to the one with two $\mathrm{O} 2^{+}$planes. This is unlike the $\mathrm{O}^{+}$case,

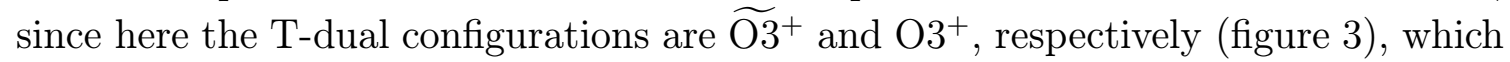
are equivalent.

In general, the third differential $d_{3}$ will not correspond to just the cup product with $H$, but the intuition remains. If a field strength $G_{n}$ can be written as a derivative $d_{m} C_{n-m}$, then it can be trivialized by a gauge transformation, and does not truly index a new RR field configuration.

\footnotetext{
${ }^{9}$ Two $\mathrm{OM}_{2}^{-}$planes correspond to an $\mathrm{O}_{2}^{-}$plane, and two $\mathrm{OM} 2^{+}$planes correspond to an $\widetilde{\mathrm{O} 2}{ }^{-}$ plane
} 


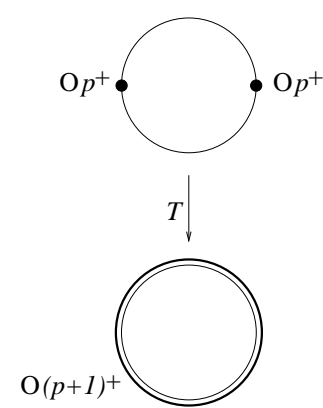

a

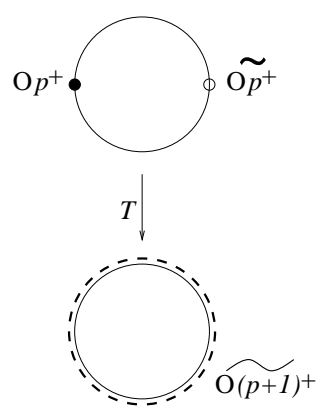

b

Figure 3: T-duality relates $(a)$ a configuration of two $\mathrm{O}^{+}$-planes at opposite points on a circle to a single $\mathrm{O}(p+1)^{+}$-plane wrapping the circle, and $(b)$ a configuration of one $\mathrm{O} p^{+}$ and one $\widetilde{\mathrm{O} p}+$ to a wrapped $\widetilde{\mathrm{O}(p+1)^{+}}$. The two configurations are equivalent for $p \leq 2$.

\subsection{Unphysical orientifolds}

For $\mathrm{O} p^{+}$planes with $p \leq 2$ there is one more modification of the AHSS due to the differential $d_{3}$. The map

$$
d_{3}: H^{2-p}\left(\mathbb{R P}^{8-p}\right) \stackrel{\cup H}{\longrightarrow} H^{5-p}\left(\mathbb{R P}^{8-p}\right)=\mathbb{Z}_{2}
$$

where the cohomologies are appropriately normal or twisted $\left(\bar{A}_{-} \overline{1} \overline{0}_{-1}^{\prime}\right)$, implies that $G_{2-p}$ is not $d_{3}$-closed, but rather

$$
d_{3} G_{2-p}=\omega_{5-p} \in \mathbb{Z}_{2} .
$$

If $\omega_{5-p}$ is the non-trivial element of $\mathbb{Z}_{2}$ then the corresponding class in $H^{2-p}$ cannot be lifted to K-theory. In particular, for the $\mathrm{O}^{+}$and $\mathrm{O}^{+}$planes $G_{2-p}$ torsion is completely obstructed, which means that the variants $\widehat{\mathrm{O}}^{+}$and $\widehat{\mathrm{O}}^{+}$(as well as the composites $\widetilde{\mathrm{O}}^{+}$and $\widetilde{\mathrm{O}}^{+}$) do not exist.

For the $\mathrm{O}_{2}^{+}$plane the above map is $\mathbb{Z} \rightarrow \mathbb{Z}_{2}$, which implies that odd values of $G_{0} / 2 \pi$ are not allowed. We will see in the next section that this can also be understood in terms of an anomaly in the three-dimensional gauge theory on D2branes in an $\mathrm{O}^{+}$background with odd $G_{0} / 2 \pi$.

It would be interesting to find a physical interpretation for these obstructions, analogous to the interpretations we gave for the equivalences before.

\section{The orientifold 6-plane}

\subsection{K-theory}

In the previous section we used the AHSS to obtain new insight into how the cohomological classification of RR fluxes in orientifold backgrounds is modified by K-theory. In particular, we saw that independent RR fluxes may become correlated, certain 
RR fluxes become trivial, and others are obstructed. For the O6-planes the latter features are absent for dimensional reasons. On the other hand we were not able to determine the correlation of the two RR fluxes $G_{2}$ and $G_{0}$.

Luckily, representative gauge bundles for the relevant K-groups, $K H\left(\mathbb{S}^{3,0}\right)$ and $K R\left(\mathbb{S}^{3,0}\right)$, can easily be worked out. Start with a flat type-IIA background, and consider complex vector bundles over $\mathbb{S}^{2}$. These are classified by $K\left(\mathbb{S}^{2}\right)=K(p t.) \oplus$ $\widetilde{K}\left(\mathbb{S}^{2}\right)=\mathbb{Z} \oplus \mathbb{Z}$, where the first term corresponds to the rank of the bundle, and the second term to its topological $\left(c h_{1}\right)$ class. Physically these correspond to D8-branes $\left(G_{0}\right.$ flux $)$ and D6-branes $\left(G_{2}\right.$ flux $)$, respectively. A convenient basis of generators to work with is $x=(1,0)$ and $y=(1,1)$. The former corresponds to a constant rank 1 bundle $v_{0}$, and the latter to the basic rank 1 (monopole) line bundle with $c h_{1}=1$. This bundle can be constructed by assigning trivial bundles $v_{+}$and $v_{-}$to the upper and lower hemishpheres, respectively, with a transition function along the equator,

$$
v_{+}(\theta)=g(\theta) v_{-}(\theta)
$$

The transition function $g(\theta)$ is a map $\mathbb{S}^{1} \rightarrow \mathrm{U}(n)$, which is classified by a winding number $w \in \pi_{1}(\mathrm{U}(n))=\mathbb{Z}$. The basic line bundle has $w=c h_{1}=1$.

Now consider the action of the involution on the two generators. On the constant bundle the action is

$$
v_{0} \longrightarrow \overline{v_{0}}
$$

if the involution squares to 1 , and

$$
v_{0} \longrightarrow \mathbb{J} \bar{v}_{0}
$$

if it squares to -1 . Consequently $x$ is a generator for $K R\left(\mathbb{S}^{3,0}\right)$, whereas $2 x$ is a generator for $K H\left(\mathbb{S}^{3,0}\right)$. The action on the line bundle is given by

$$
\begin{aligned}
& v_{+}(x) \longrightarrow \bar{v}_{-}(-x) \\
& v_{-}(x) \longrightarrow \pm \bar{v}_{+}(-x),
\end{aligned}
$$

where the upper sign corresponds to the $K R$ projection and the lower sign to the $K H$ projection. On the equator this becomes

$$
\begin{aligned}
& v_{+}(\theta) \longrightarrow \bar{v}_{-}(\theta+\pi) \\
& v_{-}(\theta) \longrightarrow \pm \bar{v}_{+}(\theta+\pi) .
\end{aligned}
$$

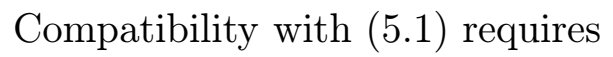

$$
g(\theta+\pi)= \pm \bar{g}(\theta)^{-1}= \pm g(\theta)^{T}
$$

which implies that $w \in 2 \mathbb{Z}$ for $K R$, and $w \in 2 \mathbb{Z}+1$ for $K H$. Thus $y$ is a generator for $K H\left(\mathbb{S}^{3,0}\right)$, whereas only $2 y$ is a generator for $K R\left(\mathbb{S}^{3,0}\right)$. 
The results are summarized as follows:

$$
\begin{array}{llll}
\text { Covering Space }: & K\left(S^{2}\right)=\mathbb{Z} \oplus \mathbb{Z} & \text { generators } x=(1,0) & y=(1,1) \\
\text { O6 }{ }^{-} \text {plane : } & K H\left(S^{3,0}\right)=\mathbb{Z} \oplus \mathbb{Z} & \text { generators } 2 x=(2,0) & y=(1,1) \\
\text { O6 }{ }^{+} \text {plane : } & K R\left(S^{3,0}\right)=\mathbb{Z} \oplus \mathbb{Z} & \text { generators } x=(1,0) & 2 y=(2,2) .
\end{array}
$$

A unit $G_{2}$ flux, i.e. a bulk D6-brane, in the reduced space corresponds to the element $2 y-2 x=(0,2)$. We therefore see that half-odd-integral $G_{2}$ flux, i.e. a fractional D6-brane, is required for $\mathrm{O}^{-}$when $G_{0}$ is odd, and forbidden when $G_{0}$ is even. In the former case the gauge group on D6-branes is $\mathrm{SO}(2 n+1)$, and we denote the orientifold plane $\widetilde{\mathrm{O}^{-}}-$. For $\mathrm{O}^{+} G_{2}$ flux is always integral, and independent of $G_{0}$. The picture is similar to the one for the lower $\mathrm{O} p$-planes, except that instead of the charge quantum being correlated with a RR torsion class (for $\mathrm{O}^{-}$), it is corellated with the parity of an integral class.

\subsection{Anomalies}

The above conclusion can also be arrived at by considering the world-volume theory on probe D-branes. It was shown in [i] that a Chern-Simons (CS) term which includes $G_{0}$ is crucial for cancellation of an anomaly with respect to large gauge transformations in the world volume theory of D2-branes in an $\widetilde{\mathrm{O}}^{-}$background. We shall review the argument, and extend it to the other cases.

Consider first a flat type-IIA background. The CS terms on a D $p$-brane ( $p$ even)

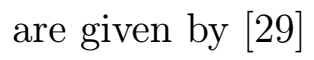

$$
S_{C S}^{f l a t}=\int_{p+1} C \wedge \operatorname{tr} e^{F / 2 \pi}-\int_{p+1} \frac{G_{0}}{2 \pi} \omega_{p+1}(A),
$$

where $\omega_{p+1}(A)$ is the Chern-Simons $(p+1)$-form defined by

$$
d \omega_{2 n-1}(A)=2 \pi \cdot \frac{1}{n !(2 \pi)^{n}} \operatorname{tr} F^{n}
$$

up to exact forms. Here 'tr' denotes the trace in the fundamental representation of the gauge group. As is well known, the integral of a CS form is not gauge invariant, but rather shifts by a $2 \pi$ multiple of the winding number of the gauge transformation. Namely, for an element $g \in \mathrm{U}(n)$ with winding $w \in \pi_{p+1}(\mathrm{U}(n))=\mathbb{Z}$, the gauge transformation $A \rightarrow g^{-1} A g+g^{-1} d g$ induces

$$
\int_{p+1} \omega_{p+1}(A) \rightarrow \int_{p+1} \omega_{p+1}(A)+2 \pi w .
$$

Invariance of the path integral therefore requires $G_{0} / 2 \pi \in \mathbb{Z}[\overline{2} \overline{2} \overline{9}]$.

In orientifold backgrounds the CS terms are given by

$$
S_{C S}=\frac{1}{2} \int_{p+1} C \wedge \operatorname{tr} e^{F / 2 \pi}-\frac{1}{2} \int_{p+1} \frac{G_{0}}{2 \pi} \omega_{p+1}(A),
$$


where now the traces are in the fundamental representation of $G=\mathrm{O}(n)$ or $\mathrm{U} \operatorname{Sp}(2 n)$, and the factor of $1 / 2$ is needed to correctly reproduce the action (15.8. $)$ when the Dbranes move away from the orientifold plane. The cases of interest are $p=2$ and 6 , as these are the only cases (in ten dimensions) with integral winding, $\pi_{3}(G)=$ $\pi_{7}(G)=\mathbb{Z}$. The integrals of the corresponding CS forms defined in (15.9.9.1) transform as follows

$$
\begin{aligned}
& \delta \int \omega_{3}(A)=\left\{\begin{array}{lll}
4 \pi w & \text { for } & \mathrm{O} \\
2 \pi w & \text { for } & \mathrm{USp}
\end{array}\right. \\
& \delta \int \omega_{7}(A)=\left\{\begin{array}{lll}
2 \pi w & \text { for } & \mathrm{O} \\
4 \pi w & \text { for } & \mathrm{USp}
\end{array}\right.
\end{aligned}
$$

where $w$ is the appropriate winding number of the gauge transformation. The relative factors of 2 can be understood by considering U Sp and $O$ as projections of $U$. The generator of $\pi_{3}(U)$ is associated with the instanton bundle on $\mathbb{S}^{4}$ (i.e. the generator of $\left.\widetilde{K}\left(\mathbb{S}^{4}\right)\right)$. Since this bundle is pseudoreal it survives the projection to U Sp, but must be doubled to get the generator for O. For $\pi_{7}$ the situation is reversed, since the associated bundle on $\mathbb{S}^{8}$ is real.

There is an additional contribution to the CS action from the fermion determinant $\operatorname{det}(i \not D)$ known as the parity anomaly. It was shown in $[\bar{\beta} \bar{\beta} \overline{0}]$ that a complex fermion in a representaion $R$ of the gauge group contributes to the three-dimensional CS term as follows

$$
S_{C S}^{1 \text {-loop }}=\frac{1}{2} \frac{m}{|m|} \int \omega_{3}^{R}(A),
$$

where $m$ is the mass of the fermion. A similar computation in seven-dimensional gauge theory gives

$$
S_{C S}^{1-\text { loop }}=\frac{1}{2} \frac{m}{|m|} \int \omega_{7}^{R}(A)
$$

Consider first $N$ D2-branes in the presence of an O6-plane and $n$ D6-branes. The case of $\mathrm{O}^{-}$was considered in [i] ${ }^{-}$. In this case the gauge group on the D2-branes is $\mathrm{USp}(2 N)$, and the global symmetry corresponding to the D6-brane gauge group is $\mathrm{O}(2 n)$ for $\mathrm{O}^{-}$and $\mathrm{O}(2 n+1)$ for $\widetilde{\mathrm{O}}^{-}$. The spectrum of (complex) fermions in the D2brane world-volume theory consists of a state in the bi-fundamental representation $(\square, \square)$, as well as a pair of states in the symmetric representation of the gauge group $(\square, \mathbf{1})$, and a pair in the antisymmetric representation $(\boxminus, \mathbf{1})$. Invariance $(\bmod 2 \pi)$ of the sum of $\left(15.11_{1}^{\prime}\right)$ and ( $\left(5,11_{1}^{\prime}\right)$ therefore requires $G_{0} / 2 \pi$ to be even for $\mathrm{O}^{-}$, and odd for $\widetilde{\mathrm{O}}^{-}$. For $\mathrm{O}^{+}$the D2-brane gauge group is $O(2 N)($ or $O(2 N+1))$, and the global symmetry is $\mathrm{U} \operatorname{Sp}(2 n)$. The fermion representations are the same as above, so in this case there is no parity anomaly. Furthermore, we see from (15.12is) that $G_{0} / 2 \pi$ can be any integer. This is exactly what we obtained in K-thoery. 
The same result can also be obtained by considering the world-volume theory on the D6-branes. In this case there is a single complex fermion in the adjoint representation of the gauge group. The CS 7-forms defined in the adjoint representation are related to those defined in the fundamental representaion by

$$
\begin{aligned}
& \int_{\Sigma_{7}} \omega_{7}^{\text {adj }}=(m-8) \int_{\Sigma_{7}} \omega_{7} \quad \text { for } \mathrm{O}(m) \\
& \int_{\Sigma_{7}} \omega_{7}^{\text {adj }}=(2 m+8) \int_{\Sigma_{7}} \omega_{7} \text { for } \quad \mathrm{USp}(2 m) .
\end{aligned}
$$

Together with $\left(15.133^{\prime}\right)$ we see that, in the first case, invariance under a large gauge transformation requires $G_{0} / 2 \pi \in 2 \mathbb{Z}$ for $\mathrm{O}^{-}$( $m$ even), and $G_{0} / 2 \pi \in 2 \mathbb{Z}+1$ for $\widetilde{\mathrm{O}}^{-}$ ( $m$ odd). There is no such correlation for $\mathrm{O}^{+}$, and $G_{0} / 2 \pi$ is an arbitrary integer.

The same approach can be applied to the O2-plane with $G_{0}$ flux, for which we only had partial K-theory results. In particular, we determined that $G_{0} / 2 \pi$ had to be even for $\mathrm{O}_{2}^{+}$, but we made no statement about $\mathrm{O}_{2}^{-}$. The world-volume theory on D2-branes in the background of an O2-plane contains an even number (four) of complex fermions in the adjoint representation in all cases, so there is no parity anomaly. Invariance under large gauge transformations therefore requires $G_{0} / 2 \pi \in 2 \mathbb{Z}$ for $\mathrm{O}^{+}$(and $\widetilde{\mathrm{O}^{2}}{ }^{+}$), but allows any integer value for $\mathrm{O}^{-}$(and $\widetilde{\mathrm{O}^{-}}{ }^{-}$). This implies, in particular, that the $G_{6}$ charge quantum is not correlated with the value of $G_{0}$ (only with the torsion class of $G_{4}$, as we saw in the previous section).

\subsection{Components of type-I vacuua}

In many ways the orientifold 6-plane was the motivation for this investigation. Compactification of type-I string theory on $\mathbb{T}^{3}$, and T-duality, yields a background with eight $\mathrm{O} 6^{-}$planes and sixteen D6-branes. On the other hand it is known that the moduli space of type-I (or heterotic) vacua on $\mathbb{T}^{3}$ has additional disconnected com-

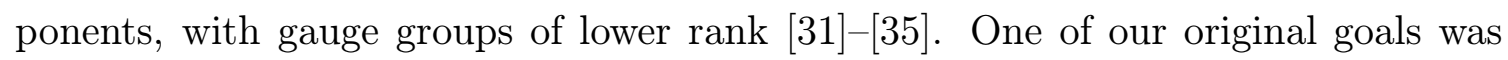
to describe these different components in the T-dual picture, using D6-branes and orientifold 6-planes.

Some of the additional components can indeed be described in this way, by including both $\mathrm{O}^{-}$and $\mathrm{O}^{+}$planes, in much the same way as one describes the eightdimensional theory without vector structure in terms of $\mathrm{O}^{-}$and $\mathrm{O}^{+}$planes [3] $\left.{ }_{3} \overline{1}_{1}\right]$. In the fully compact case the seven-dimensional orientifold contains eight O6-planes, which are located at the vertices of a cube. Since each plane can be either $\mathrm{O6}^{-}$ or $\mathrm{O} 6^{+}$, there are naively $2^{8}=256$ configurations. However not all of them are consistent, and many of them are related by the symmetry group of the 3 -torus $\mathrm{SL}(3, \mathbb{Z})$.

Consider a configuration with $N_{-} \mathrm{O} 6^{-}$planes and $N_{+} \mathrm{O} 6^{+}$planes. Supersymmetry requires $N_{-} \geq N_{+}$, since otherwise anti-D6-branes would be required for tadpole cancellation. Furthermore, consistency of the orientifold projection requires $N_{-}$and 
$N_{+}$to be even. For example, let us assume that the plane at the origin of the cube is $\mathrm{O}^{+}$, and the seven others are $\mathrm{O}^{-}$. If we denote the action of the projection on the CP factors by $\left\{g, g_{1}, g_{2}, g_{3}\right\}$, where $g_{i}$ correspond to translations along the torus, then we have

$$
\begin{aligned}
g^{T} & =-g \\
\left(g g_{i}\right)^{T} & =+g g_{i} \quad(i=1,2,3) \\
\left(g g_{i} g_{j}\right)^{T} & =+g g_{i} g_{j} \quad(i \neq j) \\
\left(g g_{1} g_{2} g_{3}\right)^{T} & =+g g_{1} g_{2} g_{3} .
\end{aligned}
$$

From the first three relations we deduce that

$$
\left\{g_{i}, g_{j}\right\}=0(i \neq j)
$$

On the other hand this implies

$$
\begin{aligned}
\left(g g_{1} g_{2} g_{3}\right)^{T} & =-\left(g g_{3} g_{2} g_{1}\right)^{T}=-g_{1}^{T} g_{2}^{T} g_{3}^{T} g^{T} \\
& =-\left(-g g_{1} g^{-1}\right)\left(-g g_{2} g^{-1} g\right)\left(-g_{3} g^{-1}\right)(-g) \\
& =-g g_{1} g_{2} g_{3}
\end{aligned}
$$

in contradiction with the last relation. The allowed values of $\left(N_{-}, N_{+}\right)$are thus $(8,0),(6,2)$, and $(4,4)$ (figure ' $\overline{4}$ ). In the first case the configuration is unique and corresponds to the original rank 16 component (figure 'isa). In the $(6,2)$ case the configuration is unique up to $\operatorname{SL}(3, \mathbb{Z})$ transformations, and the rank is 8 (figure $\operatorname{As}_{-1} b$ ). In the $(4,4)$ case there is no gauge group, and there are actually two $\operatorname{SL}(3, \mathbb{Z})$ equivalence classes. One corresponds to configurations in which four O6-planes of the same type are co-planar (figure $\underbrace{1}_{-1} \mathrm{~s} c$ ), and the other to configurations where they are not

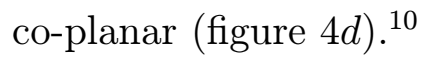

Given the previous results, we now understand that the above configurations require $G_{0} / 2 \pi$ to be even. In particular, they exist for $G_{0}=0$. For $G_{0} / 2 \pi$ odd on the other hand, we must replace $\mathrm{O}^{-}$by $\widetilde{\mathrm{O}}^{-}$, and change the number of D6-branes appropriately to cancel the tadpole. This can only be done supersymmetrically in the $(8,0)$ and $(6,2)$ configurations, which yield new components with a rank 12 and a rank 5 gauge group, respectively. (In the $(4,4)$ configurations the total charge of the $\widetilde{O 6}$-planes is +2 , and can only be cancelled by anti-D6-branes.) Of course since $G_{0} \neq 0$ in these cases, there is a non-vanishing vacuum energy, and supersymmetry is spontaneously broken.

\footnotetext{
${ }^{10}$ Since $M \in \mathrm{SL}(n, \mathbb{Z})$ satisfies $\operatorname{det} M=1$, it follows that the Levi-Civita tensor $\epsilon_{i_{1} \cdots i_{n}}$, and therefore also the pseudo-scalar product of $n$ vectors $\epsilon_{i_{1} \cdots i_{n}} v^{i_{1}} \cdots v^{i_{n}}$, is invariant under $\mathrm{SL}(n, \mathbb{Z})$. For $n=3$ this means that co-planarity is an $\mathrm{SL}(3, \mathbb{Z})$-invariant property.
} 


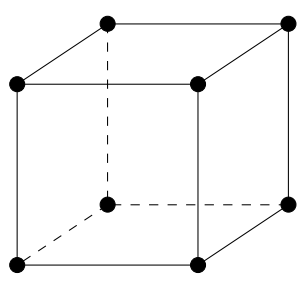

a

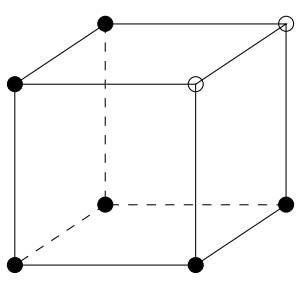

$\mathrm{b}$

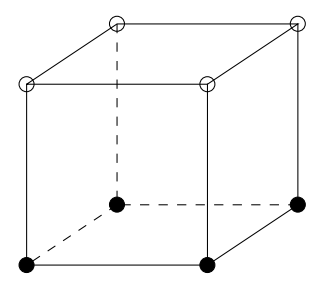

$\mathrm{c}$

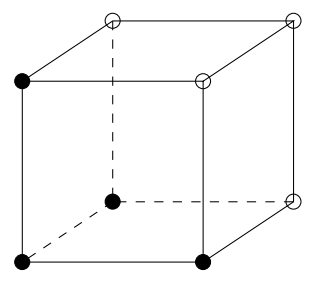

d

Figure 4: Consistent $\mathrm{SL}(3, \mathbb{Z})$-inequivalent configurations of O6-planes: $(a)$ eight $\mathrm{O}^{-}$ planes (rank 16), (b) six $\mathrm{O}^{-}$planes and two $\mathrm{O}^{+}$planes (rank 8), $(c)+(d)$ two inequivalent configurations of four $\mathrm{O}^{-}$planes and four $\mathrm{O}^{+}$planes (rank 0$)$.

\section{Conclusions and open questions}

In this paper we have demonstrated how K-theory generates very useful information on the nature of discrete RR G-fluxes in string theory, specifically for orientifold backgrounds. We saw that certain G-fluxes are correlated, that some become identified due to discrete transformations, and finally that some are disallowed outright. A very useful tool in this process was the Atiyah-Hirzebruch Spectral Sequence and our generalization of it. The AHSS ties together our understanding of separate RR-fields (even torsion ones) in terms of single K-classes.

The AHSS was particularly powerful for our set of examples because we already knew the relevant K-groups. However, use of the AHSS in general faces two obstacles. The first obstacle involves computing higher differentials in the sequence. We were able to circumvent this obstacle by using our exact knowledge of the K-groups. For

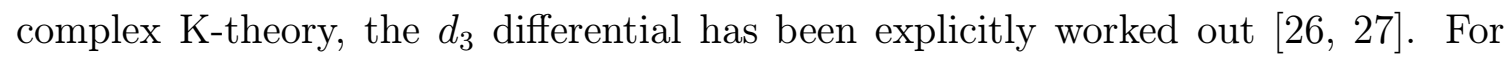

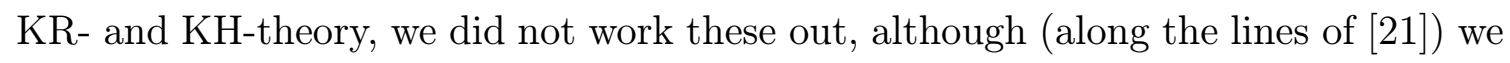
can see that adding the torsion $\mathrm{H}$-fields to $d_{3}$ brings us from one to the other. We would like to conclude by making some comments regarding the other obstacle: the matter of extensions.

One way to understand the extension problem is to realize that while the graded complex generated by the AHSS yields the elements of a K-group, it does not provide us with their additive structure. We propose that this additive structure can be extracted by thinking of the K-group elements on the asymptotic sphere as limiting values of generalized differentials in the bulk. For our purposes, a general differential form consists of a vector of differential forms of different ranks with a differential action which can increase the rank by more than one (for some ideas along these

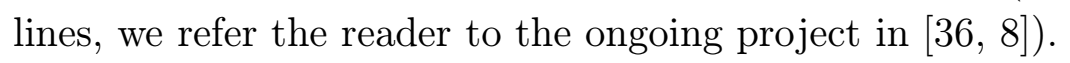

The equation of motion for fields described by generalized differentials involve the generalized derivative (naively a combination of the $d_{1}, d_{3}$ and $d_{5}$ seen in the AHSS), which in general will not be zero but can also give a K-class corresponding to the source of the particular RR-fields. The novelty here is that a K-class corresponding 
to a $p$-form current $J_{p}$ could be a source for any field strength $G_{m}$ with $m \leq p-1$, instead of just for $G_{p-1}$. Consistency (commutativity) of the additive structure with the differential action will put useful restrictions on the additive structure of the generalized differential forms, which will in turn help determine the group structure of the K-group at infinity.

Another outstanding issue concerns the absolute charges of the orientifold. In this work we have only used K-theory to compute jumps in the charges, and one can make use of the fact that K-theory contributes an integral index to the path-integral to argue that the jumps are anomaly free [i] be determined, the bare charge of the orientifold plane (for example, an $O 3^{-}$-plane

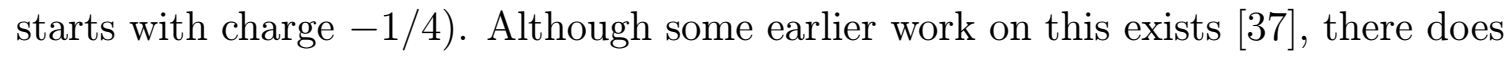
not yet exist a satifactory answer within the K-theory framework.

Note added: the classification of consistent and inequivalent O6-plane configurations (for $G_{0}=0$ ) in subsection 5.3 .3 also appeared in [3]

\section{A. AHSS in complex and real K-theory}

In this appendix we will summarize the notion of the Atiyah-Hirzebruch spectral sequence (AHSS), which can be used to compute K-theory groups which have a triangulation. We will show how this generalizes to real variants of K-theory. The first step in using the AHSS is to define an associated graded complex.

\section{A.1 Defining the associated graded complex}

Consider a space $X$, with a triangulation $\hat{X} . X_{p}$ is the subset of this triangulation with simplices of maximum dimension $p$. For example the two-sphere, $\mathbb{S}^{2}$, has a triangulation $\hat{X}=X_{2}$ equal to

$$
\left\{\left(x_{2} x_{3} x_{4}\right),\left(x_{1} x_{3} x_{4}\right),\left(x_{1} x_{2} x_{4}\right),\left(x_{1} x_{2} x_{3}\right)\right\}
$$

and so

$$
\begin{aligned}
& X_{1}=\left\{\left(x_{1} x_{2}\right),\left(x_{1} x_{3}\right),\left(x_{1} x_{4}\right),\left(x_{2} x_{3}\right),\left(x_{2} x_{4}\right),\left(x_{3} x_{4}\right)\right\} \\
& X_{0}=\left\{x_{1}, x_{2}, x_{3}, x_{4}\right\}
\end{aligned}
$$

We are now going to use the triangulation to filter the group $\mathrm{K}(\mathrm{X})$. Define

$$
K_{0}(X) \doteq K(X),\left.K_{p}(X)\right|_{1 \leq p \leq \operatorname{Dim}(X)} \doteq\left\{x \in K(X) \mid x \text { trivial on } X_{p-1}\right\} .
$$

Going back to our example $X=\mathbb{S}^{2}$, we have $K_{0}\left(\mathbb{S}^{2}\right)=\mathbb{Z} \oplus \mathbb{Z}$. When we wrap a $\mathrm{D} p$-brane on this $S_{2}$, these two charges correspond to $\mathrm{D} p$-brane charge and $\mathrm{D}(p-2)$ brane charge. Thus in this example, $K_{1}\left(\mathbb{S}^{2}\right)=K_{2}\left(\mathbb{S}^{2}\right)=\mathbb{Z}$, reflecting the fact that these groups capture only $\mathrm{D}(p-2)$-brane charge. 
For a finite dimensional space $\mathrm{X}$ of dimension $N$, the $K_{p}$ 's define a filtration,

$$
K=K_{0} \supseteq K_{1} \supseteq \cdots \supseteq K_{N}
$$

which makes $K(X)$ a filtered complex. This allows us to define an associated graded complex

$$
\mathrm{Gr} K \doteq \bigoplus_{p=0}^{p=N} K_{p} / K_{p+1} \doteq \bigoplus_{p=0}^{p=N} G_{p} .
$$

Although $\operatorname{Gr} K(X)$ doesn't capture all the structure of $K(X)$, there is, as it turns out, a simple algorithm for computing GrK which usefully connects with much of the physicist's intuition about fields as seen in cohomology. Also note that we can easily extend the notion of the associated graded complex $\operatorname{Gr} K$ to any other K-theory, such as $K^{-1}(X), K R(X), K_{ \pm}(X)$, etc.

\section{A.2 Computing $\operatorname{Gr} K$ using a spectral sequence}

To compute GrK, we are going to use a sequence of successive approximations. The first approximation to $G_{p}$ we can label as $E_{1}^{p}$, and it consists of K-classes on the $p$-simplices $\sigma_{p}^{i}$ trivial on their boundaries. In other words,

$$
E_{1}^{p}=K\left(\oplus_{i} \sigma_{i}^{p}, \oplus_{i} \dot{\sigma}_{i}^{p}\right)=\oplus_{i} K\left(\sigma_{i}^{p}, \dot{\sigma}_{i}^{p}\right) .
$$

Of course, since $K\left(\sigma_{i}^{p}, \dot{\sigma}_{i}^{p}\right)=\widetilde{K}\left(\mathbb{S}^{p}\right)=K^{-p}(p t)$ we get that

$$
E_{1}^{p}=C^{p}\left(X, K^{-p}(p t)\right)
$$

Since we our using complex K-theory for now, only the even-dimensional co-chains appear.

Now the first approximation gives us a candidate K-classes on the $\sigma_{i}^{p}$ 's, but we need to make sure that they can be extended to K-classes on $\hat{X}$ and thus $X$. The first step is to extend a class on the $p$-skeleton to a class on the $(p+1)$-skeleton, i.e. take every element in $E_{1}^{p}$ and extend it to the $\sigma_{i}^{p+1}$ 's. Summing up the integers from each component of the boundary $\dot{\sigma}_{i}^{p+1}$ yields, with appropriate orientation, a winding number which if non-zero acts as an obstruction to extending the co-chain to the $(p+1)$-skeleton. This is precisely the cohomology differential which maps

$$
d_{1}: C^{p}\left(X, K^{-p}(p t)\right) \longrightarrow C^{p+1}\left(X, K^{-p}(p t)\right)
$$

which implies that the next approximation to $G_{p}$ should be

$$
E_{2}^{p}=H^{p}\left(X, K^{-p}(p t)\right) .
$$

To continue with this construction, we define elements

$$
E_{2}^{p, q}=H^{p}\left(X, K^{q}(p t)\right) \text { with } E_{2}^{p}=E_{2}^{p,-p},
$$


where $p$ ranges from 0 to the dimension of $\mathrm{X}$, but $q$ is allowed to range freely. With these elements, the whole approximation scheme can then be refined recursively as follows. We define $d_{r}$ to be the obstruction to lifting a K-class in $E_{r}$ up $r$ steps. Formally,

$$
d_{r}: E_{r}^{p, q} \longrightarrow E_{r}^{p+r, q-(r-1)} .
$$

We then define $E_{r+1}$ as the cohomology of the operator $d_{r}$ and repeat. We have already seen what $E_{1}, d_{1}$ and $E_{2}$ correspond to. In complex K-theory, only odd differentials appear, and $d_{3}$ is the Steenrod square $S q^{3}$. For finite dimensional spaces, this procedure terminates at some finite order $n$. We then have

$$
K_{p}(X) / K_{p+1}(X)=G_{p}(X)=E_{n}^{p,-p}(X) .
$$

There are two features to this construction that are of particular interest. The first is that cohomology appears at the $E_{2}$ level in the scheme, hence we can view this construction of K-classes as a sort of refinement of $H^{*}(X)$ with a different addition structure. The second is that the same coefficients $E_{r}^{p, q}$ can naturally be used to compute the associated graded complex for other K-theories (as long as there is no involution on the space $X)$ such as $K^{-s}(X)$ and $K O^{-s}(X)$ using a slight modification

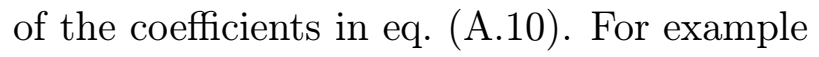

$$
G_{p}^{-s}(X) \doteq K_{p}^{-s}(X) / K_{p+1}^{-s}(X)=E_{n}^{p,-(p+s)}(X) .
$$

Note that the differentials $d_{r}$ all map from the $E^{p, q}$ 's for $K^{-s}(X)$ to those for $K^{-s+1}(X)$ just as the regular cohomology differential $d$ maps co-chains in $C^{s}(X)$ to co-chains in $C^{s+1}(X)$.

\section{A.3 Extending the AHSS to K-theories with freely acting involutions}

In order to extend the AHSS construction above to K-theories with a freely acting spatial involution, e.g. $K R^{-s}\left(\mathbb{S}^{p, 0}\right)$, we need to make some restrictions on the triangulation, namely that every simplex $\sigma_{i}^{p}$ is mapped by the involution $\chi$ to another simplex ${\overline{\sigma_{i}}}^{p}$ such that

$$
\sigma_{i}^{p} \cap{\overline{\sigma_{i}}}^{p}=\emptyset
$$

As an example, we can give a simple such triangulation for the $\mathbb{S}^{p, 0}$. Start with the set of points $\left\{x_{ \pm}^{1}, \ldots, x_{ \pm}^{p}\right\}$. The simplices that make up the triangulation consist of all sets $S$ of these points (with appropriate orientations) such that

$$
x_{ \pm}^{i} \in S \Rightarrow x_{\mp}^{i} \notin S .
$$

For example, $\left(x_{+}^{1} x_{-}^{2} x_{+}^{3}\right)$ is a 2 -simplex in this triangulation, but $\left(x_{+}^{1} x_{-}^{2} x_{-}^{1}\right)$ is not. We then define the involution to to take

$$
\chi:\left(x_{ \pm}^{i}, \ldots, x_{ \pm}^{j}\right) \longrightarrow\left(x_{\mp}^{i}, \ldots, x_{\mp}^{j}\right) .
$$

which clearly satisfies eq. (1A 
Given a proper triangulation then, we can continue to follow the procedure above, except now there should be a slight modification of the $E_{1}$ term given in eq. ( For example, for computing $K R$-groups we use

$$
E_{1}^{p}=K R\left(\oplus_{i}\left(\sigma_{i}^{p} \oplus \bar{\sigma}_{i}^{p}\right), \oplus_{i}\left(\dot{\sigma}_{i}^{p} \oplus \dot{\bar{\sigma}}_{i}^{p}\right)\right)=\oplus_{i} K R\left(\sigma_{i}^{p} \oplus \bar{\sigma}_{i}^{p}, \dot{\sigma}_{i}^{p} \oplus \dot{\bar{\sigma}}_{i}^{p}\right)
$$

At this point it useful to bear in mind that generator of $K\left(\sigma^{i}, \dot{\sigma}^{i}\right)$ is real (or pseudo real) for $i=0 \bmod 4$ but not for $i=2 \bmod 4$ (the conjugate spinors have opposite chirality) . This implies that for $K R$ - and $K H$-groups,

$$
\begin{gathered}
E_{1}^{p, q}=C^{p}\left(\left.X\right|_{\chi}, \mathbb{Z}\right) \text { for } q=0 \bmod 4 \\
E_{1}^{p, q}=C^{p}\left(\left.X\right|_{\chi}, \widetilde{\mathbb{Z}}\right) \text { for } q=2 \bmod 4 \\
E_{1}^{p, q}=0 \text { for } q \text { odd }
\end{gathered}
$$

where $\widetilde{Z}$ refers to the local coefficients on $\mathrm{X}$ which switch signs with the map $\chi$, i.e. the co-chain takes opposite value for opposite simplices in the covering space $X$.

The differential $d_{1}$ is identical with the usual differential operator, so $E_{2}$ gives twisted and un-twisted cohomologies. On the other hand, we can see from eq. ('A. $1 \overline{1}$ is that $d_{3}$ takes twisted chains to un-twisted chains and vice-versa and so is not the Steenrod square like in the complex case. It provides for a difference between KRand KH-groups.

\section{A.4 Illustrative example: $K R\left(\mathbb{S}^{6,0}\right)$ and $K H\left(\mathbb{S}^{6,0}\right)$}

To illustrate the AHSS in the context of KR-groups and KH-groups, we can examine as an example the groups $K R\left(\mathbb{S}^{6,0}\right)$ and $K H\left(\mathbb{S}^{6,0}\right)$. The $E_{2}$ terms are:

$$
\begin{aligned}
E_{2}^{0,0}\left(\mathbb{S}^{6,0}\right) & =H^{0}\left(\mathbb{R} P^{5}, \mathbb{Z}\right)=\mathbb{Z} \\
E_{2}^{1,-1}\left(\mathbb{S}^{6,0}\right) & =0 \\
E_{2}^{2,-2}\left(\mathbb{S}^{6,0}\right) & =H^{2}\left(\mathbb{R} P^{5}, \widetilde{\mathbb{Z}}\right)=0 \\
E_{2}^{3,-3}\left(\mathbb{S}^{6,0}\right) & =0 \\
E_{2}^{4,-4}\left(\mathbb{S}^{6,0}\right) & =H^{4}\left(\mathbb{R} P^{5}, \mathbb{Z}\right)=\mathbb{Z}_{2} \\
E_{2}^{5,-5}\left(\mathbb{S}^{6,0}\right) & =0 .
\end{aligned}
$$

There are two possible places where the differential $d_{3}$ can act non-trivially:

$$
\begin{gathered}
d_{3}: E_{2}^{0,0}\left(\mathbb{S}^{6,0}\right)=\mathbb{Z} \rightarrow E_{2}^{3,-2}\left(\mathbb{S}^{6,0}\right)=\mathbb{Z}_{2} \\
d_{3}: E_{2}^{1,-2}\left(\mathbb{S}^{6,0}\right)=\mathbb{Z}_{2} \rightarrow E_{2}^{4,-4}\left(\mathbb{S}^{6,0}\right)=\mathbb{Z}_{2} .
\end{gathered}
$$

Let us look at the case $\left(\bar{A}_{-}^{-} \overline{2} \bar{i}\right)$ more closely. The generator of $E_{2}^{0,0}\left(\mathbb{S}^{6,0}\right)$ is a bundle of rank one over the 0 -skeleton. Being in $E_{2}$ (and thus $E_{3}$ ) means that it extends to a rank one bundle over the 2-skeleton. Let's try to extend it to a rank 
one bundle over the 3 -skeleton. Consider the 3 -chain in $C_{3}\left(\mathbb{R P}^{5}, \widetilde{\mathbb{Z}}\right)$ which looks like an $\mathbb{R P}^{3}$ inside $\mathbb{R} \mathrm{P}^{5}$. It has as a boundary a 2-chain made up of two $\mathbb{R} \mathrm{P}^{2}$ 's (which explains why $\left.H_{3}\left(\mathbb{R P}^{5}, \widetilde{\mathbb{Z}}\right)=0\right)$. In KR-theory, the constant rank one bundle over the $\mathbb{R P}^{2}$ 's can be extended to the " $\mathbb{R P}$ ", , but in KH-theory the rank one bundle over $\mathbb{R} \mathrm{P}^{2}$ has non-trivial first Chern class and so cannot be extended to the " $\mathbb{R} \mathrm{P}^{3}$ ". Looking at eq. ( $(1.22)$, this fact implies that $d_{3}$ takes $z \rightarrow 0$ in $K R\left(\mathbb{S}^{6,0}\right)$ but that $z \rightarrow z \bmod 2$ in $K H\left(\mathbb{S}^{6,0}\right)$. Further analysis of the same kind reveals that $d_{3}$ is only non-trivial in eq. ( $\left(\bar{A}^{-} . \overline{2} \overline{3}\right)$ for $K H\left(\mathbb{S}^{6,0}\right)$. The final result is then:

$$
\begin{aligned}
& K R\left(\mathbb{S}^{6,0}\right)=\mathbb{Z} \oplus \mathbb{Z}_{2} \\
& K H\left(\mathbb{S}^{6,0}\right)=2 \mathbb{Z} .
\end{aligned}
$$

where the factor of 2 denotes the fact that only even rank constant bundles are allowed.

\section{Acknowledgments}

O.B. and E.G. would like to thank M. Atiyah, A. Hanany, B. Kol, and especially E. Witten for useful conversations. S.S. would like to thank Y. Hyakutake and Y. Imamura for useful discussions. O.B. was supported in part by the DOE under grant no. DE-FG03-92-ER40701, and by a Sherman Fairchild Prize Fellowship. The work of E.G. was supported by the NSF under grant no. 0070928 and Frank and Peggy Taplin. The work of S.S. was supported in part by the Japan Society for the Promotion of Science.

\section{References}

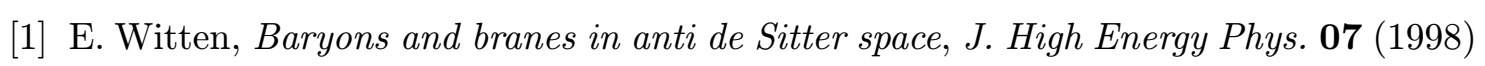
1.

[2] A. Hanany and B. Kol, On orientifolds, discrete torsion, branes and $M$ theory, ${ }_{1}^{\prime} \bar{H}_{-} \bar{H} i \bar{g} \bar{h}_{1}^{\prime}$

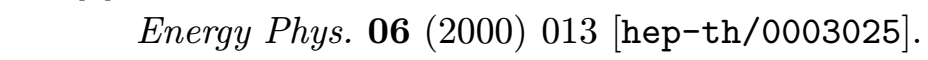

[3] E. Witten, On flux quantization in M-theory and the effective action, J. Geom. Phys. 22 (1997) 1-13 [hep-th/9609122].

[4] R. Minasian and G. Moore, K-theory and Ramond-Ramond charge, iJ. $\bar{H} \bar{i} \bar{g} \mathrm{E}$ Energy. [

[5] E. Witten, D-branes and K-theory, iJ. High Energy Phys. 12 $(1 \overline{1} 9 \overline{9})^{-} 01 \overline{1}$ [hep-th/981018醇.

[6] P. Hořava, Type-IIA D-branes, K-theory and matrix theory, iAdv. Theor. Máth. i. 


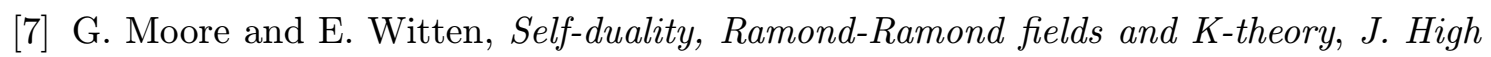

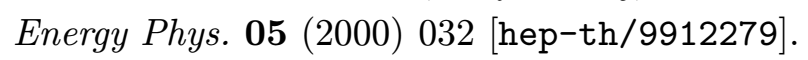

[8] D.S. Freed and M.J. Hopkins, On Ramond-Ramond fields and K-theory, 'J.

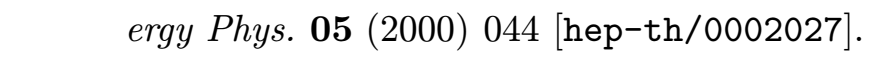

[9] E.G. Gimon and J. Polchinski, Consistency conditions for orientifolds and Dmanifolds, 'Phys. Rev. D 54 (1996) 1667' [hep-th/9601038'].

[10] S. Elitzur, A. Giveon, D. Kutasov and D. Tsabar, Branes, orientifolds and chiral gauge

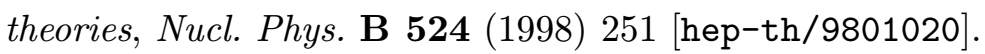

[11] S. Sethi, A relation between $N=8$ gauge theories in three dimensions,

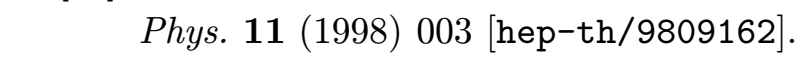

[12] E.G. Gimon, On the M-theory interpretation of orientifold planes, hiep-th/98062 $2 \overline{6}_{.}^{\prime}$

[13] K. Hori, Consistency condition for fivebrane in $M$-theory on $\mathbb{R}^{5} / \mathbb{Z}_{2}$ orbifold, iNيuicl: :

[14] K. Landsteiner, E. Lopez and D.A. Lowe, $N=2$ supersymmetric gauge theories,

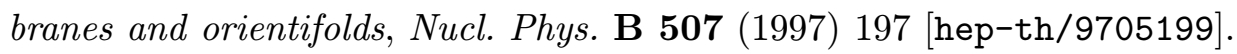

[15] L.J. Romans, Massive $N=2 a$ supergravity in ten-dimensions, 'Phys. Lett. :

[16] J. Polchinski, Dirichlet-branes and Ramond-Ramond charges, 'Phhys. Rev. Lett. :-

[17] Y. Hyakutake, Y. Imamura and S. Sugimoto, Orientifold planes, type I Wilson lines and non-BPS D-branes, iJ. High Energy Phys. ō

[18] N. Evans, C.V. Johnson and A.D. Shapere, Orientifolds, branes and duality of $4 D$ gauge theories, Nucl. Phys. B 505 (1997) 251; [hep-th/9703210i].

[19] C.V. Johnson, On the orientifolding of type II NS-fivebranes, 'P $\bar{h} \bar{h} \bar{s}$. .

[20] O. Aharony and A. Rajaraman, String theory duals for mass-deformed $\mathrm{SO}(N)$ and

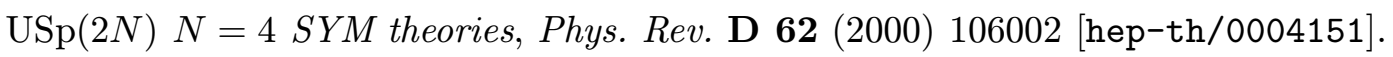

[21] D.S. Freed and E. Witten, Anomalies in string theory with D-branes, hepe-th/9907189.

[22] M. Atiyah, K-theory and reality, reprinted in Michael Atiyah collected works, vol. 2, K-theory, Clarendon Press, Oxford 1988.

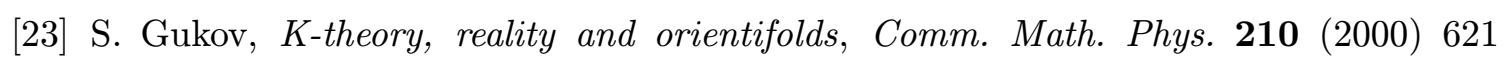
hep-th/9901042in.

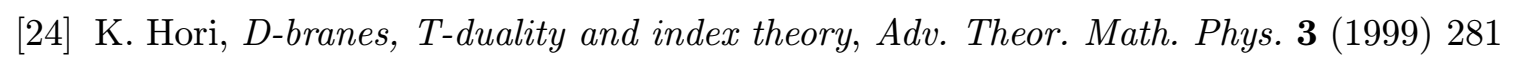
[hep-th/9902102i]. 
[25] O. Bergman, E.G. Gimon and P. Hořava, Brane transfer operations and T-duality of

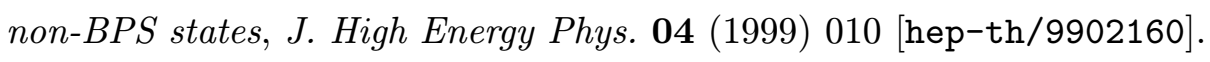

[26] M.F. Atiyah and F. Hirzebruch, Vector bundles and homogeneous spaces, Proc. Sympos. Pure Math., vol. III, pp 7-38, AMS, Providence 1961.

[27] D.-E. Diaconescu, G. Moore and E. Witten, E(8) gauge theory and a derivation of $K$-theory from $M$-theory, hep-thy0005090'.

[28] O. Bergman, E. Gimon and B. Kol, Strings on orbifold lines, .

[29] M.B. Green, C.M. Hull and P.K. Townsend, D-brane Wess-Zumino actions, T-duality and the cosmological constant, Phys. Lett. B $\mathbf{3} 8 \mathbf{2}(1996) 65$

[30] A.N. Redlich, Parity violation and gauge noninvariance of the effective gauge field

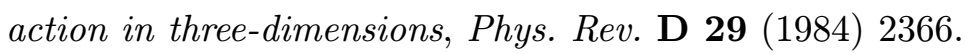

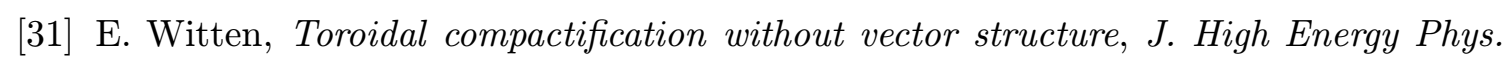
:

[32] A. Keurentjes, A. Rosly and A.V. Smilga, Isolated vacua in supersymmetric Yang-

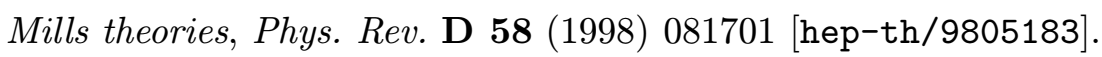

[33] A. Keurentjes, Non-trivial flat connections on the 3-torus I: G(2) and the orthogonal groups, nections on the 3-torus II: the exceptional groups $F(4)$ and $E(6,7,8)$, iJ ${ }_{-}$High Energy

:- - Phys. 05 (1999) 014: [hep-th/9902186]; Orientifolds and twisted boundary conditions,

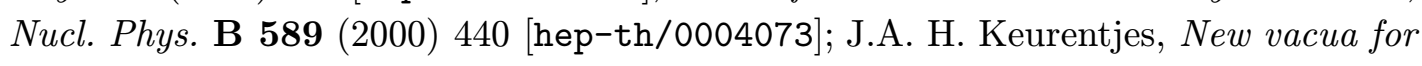
Yang-Mills theory on a 3-torus, hep-th/0007196.

[34] V.G. Kac and A.V. Smilga, Vacuum structure in supersymmetric Yang-Mills theories with any gauge group, hep-th/9902029.

[35] A. Borel, R. Friedman and J. W. Morgan, Almost commuting elements in compact Lie groups, math. gr 19907007 .

[36] D.S. Freed, Dirac charge quantization and generalized differential cohomology, hep-th/0011220;

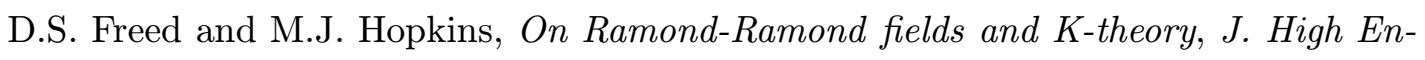

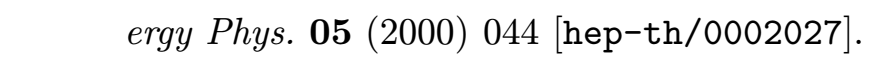

[37] K. Dasgupta, D.P. Jatkar and S. Mukhi, Gravitational couplings and $\mathbb{Z}_{2}$ orientifolds,

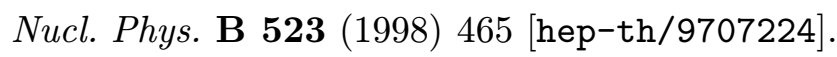

[38] J. de Boer et al., Triples, fluxes and strings, hes-th/0103170̄.'. 